\title{
Construcción y validez de la Batería Neuropsicológica Digital Infantil (BANEDI)
}

\section{Design and validity of the digital battery of developmental neuropsychological assessment (BANEDI)}

\author{
Alexa von Hagen, Sandra Berta, Sofía Morel, Rosina Perrier, María Elena Brenlla y Denise \\ del Arca
}

\begin{abstract}
Nota de autor
Alexa von Hagen, Goethe University Frankfurt, Alemania; Sandra Berta, Virtualway SRL, Uruguay; Sofía Morel, Centro Hospitalario Pereira Rossell, Uruguay; Rosina Perrier, Virtualway SRL, Uruguay; María Elena Brenlla, Centro de Investigaciones en Psicología y Psicopedagogía, Universidad Católica Argentina, Argentina; Denise del Arca, Universidad Católica del Uruguay, Uruguay.

Toda correspondencia relacionada con este artículo debe ser enviada a Sandra Berta, Virtualway SRL, Juan Benito Blanco 990 apto 101, CP 11300, Montevideo, Uruguay. Correo electrónico: sbertal@gmail.com
\end{abstract}

\section{Agradecimientos}

Agradecemos el apoyo financiero y profesional de la Agencia Nacional de Investigación e Innovación (ANII) del Uruguay por medio del programa Implementación de la innovación para posibilitar la elaboración de la Batería Neuropsicológica Digital Infantil (BANEDI). A su vez agradecemos a todo el equipo de trabajo que participó en la elaboración de la BANEDI: Valeria Abusamra, Lucía Acle, Inés Acosta, Noemí Boado, Victoria Bonino, Luciana Buonsati, Ariel Cuadro, Lucas Damiani, Sergio Dansilio, Pablo Duque, Magdalena Folgado, Liliana Fonseca, Valeria Fraga, Leticia Gómez, Bárbara Gottheil, Marcelo Inverso, Verónica Maggio, Griselda Mapeu, María José Mizraji, Valeria Oddone, Santiago Pereda, Paola Pitta, María Saravia, Cristina Scavone, Mariana Seivane, ZeppelinLabs, DAR Soluciones, Mirazoqui Estudio, todos los profesionales y estudiantes avanzados que realizaron la recolección de datos en Uruguay y Argentina, así como todos los docentes, padres y alumnos que participaron de este proyecto. 


\section{Resumen}

Este reporte presenta información sobre el proceso de construcción y evidencia de validez de la Batería Neuropsicológica Digital Infantil (BANEDI) en base a los datos recolectados de una muestra de 988 niños/adolescentes de Montevideo, Uruguay y Buenos Aires, Argentina. Enmarcado en aportes recientes de la neuropsicología cognitiva, BANEDI permite evaluar el desarrollo neuropsicológico de niños y adolescentes de 4 a 15 años y cursando Educación Preescolar Nivel 4 a $3^{\circ}$ de Educación Secundaria en la región del Río de la Plata. Apunta a ser un recurso de utilidad clínica y rigor científico para recolectar información sobre los siguientes ocho dominios cognitivos: memoria, atención y funciones ejecutivas, teoría de la mente, habilidades metalingüísticas, lenguaje oral, habilidades visoespaciales, lenguaje escrito y habilidades matemáticas. Consta de un total de 75 pruebas y dos cuestionarios para padres y docentes.

En este artículo proveemos información sobre los resultados de análisis de parametrización de los ítems de las pruebas, así como evidencia de la validez de contenido, de criterio y evolutiva de la BANEDI. En conclusión, los resultados reflejan que la BANEDI cumple con los criterios de calidad psicométrica necesarios para realizar una estimación válida del funcionamiento neuropsicológico de niños/adolescentes entre 4 y 15 años de edad y cursando Educación Preescolar Nivel 4 a $3^{\circ}$ de Educación Secundaria en la región del Río de la Plata.

Palabras claves: test neuropsicológico, trastorno del desarrollo, niños, adolescentes, evaluación 


\begin{abstract}
This report presents information on the design and validity of the Batería Neuropsicológica Digital Infantil (BANEDI - in English Digital Developmental Neuropsychological Test Battery) based on data collected from a sample of 988 children/adolescents in Montevideo, Uruguay and Buenos Aires, Argentina. Building on recent contributions from cognitive neuropsychology, this instrument aims to assess the neuropsychological development of children and adolescents aged 4 to 15 years and attending Preschool to 3rd year of Secondary school in the region of Río de la Plata. BANEDI intends to be a clinically useful and scientifically rigorous tool to collect information on the following eight cognitive domains: memory, attention and executive functions, theory of mind, metalinguistic skills, oral language, visual-spatial skills, written language and mathematical skills. It consists of a total of 75 subtests and two questionnaires for parents and teachers.

In this report we provide information on the results of item parameter analyses of the subtests included in the battery, as well as evidence on the content, criterion and developmental validity of BANEDI. To conclude, results reflect that BANEDI meets the criteria to ensure psychometric quality and can therefore be used to complete a valid estimation of the neuropsychological functioning of children/adolescents aged 4 to 15 years and attending Preschool to 3rd year of Secondary school.

Keywords: neuropsychological test, developmental disorder, children, adolescents, assessment
\end{abstract}




\section{Introducción}

Los primeros registros de los orígenes de la neuropsicología en Latinoamérica se remontan al año 1958, cuando Carlos Mendilaharsu y Selika de Mendilaharsu crearon el Laboratorio de Afecciones Cerebro-Corticales en el Instituto de Neurología de Montevideo, Uruguay (Arango-Lasprilla et al., 2016; Dansilio, 2009). Si bien se destacan muchos logros y avances de la disciplina desde estos inicios aún restan muchos obstáculos por resolver. Uno de los problemas centrales refiere a la disponibilidad de instrumentos de evaluación neuropsicológica en nuestro medio. Así por ejemplo, en una encuesta realizada por ArangoLasprilla et al. (2016) con 808 profesionales radicados en Latinoamérica, que se autoidentificaron como neuropsicólogos o profesionales de la salud dedicados al campo de la neuropsicología, un 35.0\% de la muestra expresó que la ausencia de instrumentos de evaluación e intervención neuropsicológica era un problema central para el desarrollo de la disciplina. Con respecto a los instrumentos de evaluación empleados, solamente un $15 \%$ de la muestra indicó aplicar baterías estandarizadas, mientras el resto de los participantes expresó recurrir a baterías personalizadas y flexibles. Los mayores problemas señalados en relación al empleo de instrumentos de evaluación neuropsicológica fueron la ausencia de datos normativos para la población local (62\%), la falta de adaptación del instrumento a la cultura de la población objetivo (56\%) y el costo excesivo de los instrumentos (49\%).

También en el campo de la neuropsicología del desarrollo son pocos los instrumentos adaptados al español, que cuentan con datos normativos para niños y adolescentes en Latinoamérica. Una de las únicas herramientas de evaluación neuropsicológica global validada con niños y adolescentes mexicanos y colombianos empleada con mucha frecuencia en nuestro medio es la batería de Evaluación neurológica infantil - ENI-2 (Matute, Rosselli, Ardila \& Ostrosky, 2007). Por lo contrario, muchas otras herramientas de uso frecuente en Latinoamérica como la Batería Neuropsicológica infantil NEPSY-II (Korkman, Kirk \& Kemp, 2007) solamente cuentan con baremos basados en poblaciones de España o Estados Unidos. Para sobrellevar esta limitación, muchos profesionales recurren a instrumentos que cuentan con baremos locales, como por ejemplo el Test de lectura y escritura en español LEE (Defior et al., 2006) y el Test para la evaluación del procesamiento del número y el cálculo en niños Procálculo (Feld, Taussik \& Azaretto, 2006) con baremos para Argentina. Sin embargo, estas herramientas se limitan a proveer información parcial sobre determinadas funciones neuropsicológicas (p.ej. las habilidades de lenguaje escrito), pero no son útiles para realizar una evaluación global del funcionamiento neuropsicológico del niño/adolescente, que 
permita identificar fortalezas y debilidades en un amplio rango de funciones neuropsicológicas.

Además de la escasez de baremos para Latinoamérica, otro aspecto problemático es la fecha del diseño inicial y de las últimas revisiones de muchos de los instrumentos de evaluación con los que contamos en Latinoamérica. Tomando en cuenta los cambios tecnológicos en las metodologías de investigación en el campo de las neurociencias, no queda claro hasta qué punto estos instrumentos aún están basados en la evidencia científica actualizada. También los avances tecnológicos en la administración, corrección e interpretación de test psicológicos visibles en otras partes del mundo a la fecha aún no han sido incorporados en la mayoría de los instrumentos de evaluación neuropsicológica disponibles en nuestro medio. Así por ejemplo la Batería de los procesos lectores - revisada PROLEC-R (Cuetos, Rodríguez, Ruano \& Arribas, 2014), el CELF-4 Clinical Evaluation of Language Fundamentals - Spanish Edition (Semel, Wiig \& Secord, 2006), el Test de memoria y aprendizaje TOMAL (Reynolds \& Bigler, 2001) o el Test de competencia matemática básica TEMA 3 (Ginsburg, Baroody, del Río \& Guerra, 2007) todavía no cuentan con el apoyo de recursos digitales para presentar estímulos, medir tiempos de reacción, realizar correcciones automáticas y facilitar el análisis e informe de resultados. Estas innovaciones no solamente facilitan la labor del profesional en gran medida, sino que también permiten una presentación más atractiva y ecológicamente válida de las pruebas para niños y adolescentes nativos digitales.

En su conjunto, todas estas limitaciones representan obstáculos a la evaluación neuropsicológica de niños y adolescentes en nuestro medio. Con el fin de responder a algunos de los problemas nombrados anteriormente, nos propusimos como objetivo general elaborar un instrumento de utilidad clínica y rigor científico para evaluar el desarrollo neuropsicológico de niños y adolescentes de 4 a 15 años en la región del Río de la Plata (Montevideo, Uruguay y Buenos Aires, Argentina). A su vez, nos planteamos los siguientes objetivos específicos:

- poner a disposición una herramienta de evaluación neuropsicológica basada en evidencia científica actualizada,

- computar baremos regionales, que permitan la comparación de los resultados del niño/adolescente con una población normotípica adecuada,

- cumplir con criterios de rigurosidad metodológica y científica en la elaboración de test psicológicos, 
- ofrecer medios para realizar una evaluación global, que permita identificar fortalezas y debilidades en un amplio rango de funciones neuropsicológicas del niño/adolescente,

- elaborar una prueba con un diseño atractivo para el niño y el adolescente, que facilite un contacto empático y receptivo,

- presentar un manual teórico y de aplicación, que asista al profesional en la identificación de déficit en el funcionamiento neuropsicológico del niño/adolescente,

- proporcionar un instrumento digital, que facilite la labor del profesional a la hora de administrar la prueba, corregir los resultados y escribir el informe de evaluación.

En esta primera publicación científica sobre la BANEDI apuntamos a comunicar los resultados del proceso de construcción y análisis de validez del instrumento. Más específicamente profundizamos en (1) los resultados de los análisis de parametrización de los ítems de las pruebas incluidas en BANEDI y (2) en la evidencia de validez de contenido, de criterio y evolutiva de la herramienta. Si bien planificamos comunicar más resultados sobre análisis psicométricos complementarios (p.ej. evidencia de confiabilidad) en futuras publicaciones científicas, el lector interesado puede profundizar en estos aspectos desde ya en el capítulo 2 del manual teórico y de aplicación de la BANEDI en el que detallamos la justificación psicométrica completa del instrumento (ver https://osf.io/c6vqp/).

\section{Método}

\subsection{Participantes}

La muestra de tipificación de la BANEDI incluyó 988 alumnos de diez y 13 colegios privados de Montevideo, Uruguay y Buenos Aires, Argentina, respectivamente. Debido a limitaciones prácticas para acceder a los permisos pertinentes, lamentablemente no nos fue posible acceder a escuelas y liceos públicos. Con el fin de superar esta limitación y asegurar una muestra de tipificación que también fuera representativa para niños/adolescentes de niveles socio-económicos vulnerables, invitamos a instituciones educativas de dos tipos de barrios y comunas de Montevideo y de la ciudad de Buenos Aires a participar en este estudio. Para esta selección seguimos los criterios determinados por la sexta autora de este artículo descritos a continuación.

Por un lado, contactamos a colegios de los barrios o comunas más vulnerables de cada ciudad, que contaban con el mayor porcentaje de hogares con Necesidades Básicas Insatisfechas (NBI) según los datos del Censo de Población, Viviendas y Hogares del año 2011 para Uruguay y el Censo Nacional de Población, Hogares y Viviendas del año 2010 
(INEC, 2012) para Argentina. En el caso de Montevideo, de un total de diez instituciones educativas que finalmente aceptaron participar en este estudio, cuatro de ellas se situaban en barrios de este tipo y según Calvo et al. (2013 - Mapa 3, p.34) contaban con un 40.4 a $60.1 \%$ de hogares con al menos una NBI. En forma análoga, en el caso de la ciudad de Buenos Aires siete de las trece instituciones educativas incluidas en la muestra de tipificación estaban localizadas en comunas con el mayor porcentaje de hogares con NBI (11.9 a 15.9\%) según el informe de la Dirección Nacional de Relaciones Económicas con las Provincias (DINREP, 2014 - p.24). La mayoría de los colegios incluidos en esta categoría recibían apoyo financiero de organizaciones no gubernamentales y apuntaban específicamente a proveer educación a niños y adolescentes de niveles socio-económicos extremadamente descendidos.

Por otro lado, contactamos a colegios situadas en barrios y comunas con porcentajes menores de NBI, en comparación a lo descrito anteriormente. En Montevideo, seis de los diez colegios participantes cayeron en esta categoría de barrios con porcentajes de hogares con NBI menores a 40.4\% (Calvo et al., 2013 - Mapa 3, p.34). Para la ciudad de Buenos Aires, incluimos seis sobre un total de trece colegios, que estaban localizados en comunas con un porcentaje de hogares con NBI menores a 11.9\% (DINREP, 2014 - p.24).

Finalmente, del total de 23 colegios participantes, 988 niños/adolescentes de Montevideo $(n=517)$ y de la ciudad de Buenos Aires $(n=471)$ participaron del estudio de tipificación de la BANEDI. En la selección de la muestra tomamos en cuenta el sexo, la edad y el año escolar de los niños/adolescentes. Estos datos se reflejan en las Tablas 1 y 2. 


\section{Tabla 1}

Muestra de niños/adolescentes por localidad, edad y sexo

\begin{tabular}{lccccccc}
\hline \multirow{2}{*}{ Edad } & \multicolumn{2}{c}{ Montevideo } & \multicolumn{2}{c}{ Buenos Aires } & \multicolumn{2}{c}{ Total } & \multirow{2}{*}{ Total } \\
\cline { 2 - 6 } & Mujeres & Varones & Mujeres & Varones & Mujeres & Varones & \\
\hline 4 años & 29 & 27 & 21 & 19 & 50 & 46 & 96 \\
5 años & 30 & 29 & 24 & 23 & 54 & 52 & 106 \\
6 años & 31 & 27 & 24 & 22 & 55 & 49 & 104 \\
7 años & 24 & 22 & 23 & 22 & 47 & 44 & 91 \\
8 años & 29 & 21 & 24 & 23 & 53 & 44 & 97 \\
9 años & 24 & 25 & 23 & 25 & 47 & 50 & 97 \\
10 años & 30 & 28 & 22 & 20 & 52 & 48 & 100 \\
11 años & 27 & 21 & 22 & 22 & 49 & 43 & 92 \\
12 años & 17 & 13 & 22 & 19 & 39 & 32 & 71 \\
13 años & 15 & 13 & 19 & 17 & 34 & 30 & 64 \\
14 años & 16 & 11 & 17 & 16 & 33 & 27 & 60 \\
15 años & 3 & 5 & 0 & 2 & 3 & 7 & 10 \\
Subtotal & 275 & 242 & 241 & 230 & 516 & 472 & 988 \\
Total & 517 & \multicolumn{7}{c}{471} & & 988 & \\
\hline
\end{tabular}

\section{Tabla 2}

Muestra de niños/adolescentes por localidad, año escolar y sexo

\begin{tabular}{|c|c|c|c|c|c|c|c|}
\hline \multirow{2}{*}{ Año escolar } & \multicolumn{2}{|c|}{ Montevideo } & \multicolumn{2}{|c|}{ Buenos Aires } & \multicolumn{2}{|c|}{ Total } & \multirow{2}{*}{ Total } \\
\hline & Mujeres & Varones & Mujeres & Varones & Mujeres & Varones & \\
\hline Preescolar 4 & 37 & 35 & 25 & 20 & 62 & 55 & 117 \\
\hline Preescolar 5 & 30 & 29 & 23 & 25 & 53 & 54 & 107 \\
\hline $1^{\circ}$ Primaria & 36 & 27 & 24 & 22 & 60 & 49 & 109 \\
\hline $2^{\circ}$ Primaria & 18 & 18 & 24 & 20 & 42 & 38 & 80 \\
\hline $3^{\circ}$ Primaria & 32 & 24 & 21 & 27 & 53 & 51 & 104 \\
\hline $4^{\circ}$ Primaria & 24 & 27 & 26 & 20 & 50 & 47 & 97 \\
\hline $5^{\circ}$ Primaria & 31 & 24 & 22 & 21 & 53 & 45 & 98 \\
\hline $6^{\circ}$ Primaria & 22 & 16 & 24 & 25 & 46 & 41 & 87 \\
\hline $1^{\circ}$ Secundaria & 14 & 18 & 20 & 20 & 34 & 38 & 72 \\
\hline $2^{\circ}$ Secundaria & 16 & 11 & 16 & 13 & 32 & 24 & 56 \\
\hline $3^{\circ}$ Secundaria & 15 & 13 & 16 & 17 & 31 & 30 & 61 \\
\hline Subtotal & 275 & 242 & 241 & 230 & 516 & 472 & 988 \\
\hline Total & \multicolumn{2}{|c|}{517} & \multicolumn{2}{|c|}{471} & \multicolumn{2}{|c|}{988} & \\
\hline
\end{tabular}




\subsection{Materiales}

La BANEDI está compuesta por una guía para la entrevista inicial, cuestionarios y pruebas que brindan información sobre el funcionamiento comportamental, emocional, sensorial y neuropsicológico de niños y adolescentes. En relación a los cuestionarios se incluye: (1) un cuestionario de detección de síntomas afectivos y comportamentales y (2) un cuestionario de integración sensorial para recolectar información de los padres y docentes del niño/adolescente a evaluar. Las 75 pruebas apuntan a evaluar diferentes subcomponentes de los siguientes ocho dominios de funcionamiento neuropsicológico: (1) Memoria, (2) Atención y Funciones ejecutivas, (3) Teoría de la Mente, (4) Habilidades metalingüísticas, (5) Lenguaje oral, (6) Habilidades viso-espaciales, (7) Lenguaje escrito y (8) Habilidades matemáticas.

La aplicación de los cuestionarios y la mayoría de las pruebas se rigen por el criterio de la edad del niño/adolescente (4 a 15 años). Sin embargo, para algunas pruebas tomamos el año escolar que el niño/adolescente cursaba (Preescolar nivel 4 años a $3^{\circ}$ Secundaria) como criterio de aplicación para contemplar la incidencia de la instrucción formal en el desarrollo neuropsicológico del niño/adolescente. Esto resulta especialmente importante en dominios en los que la experiencia escolar tiene una alta incidencia (p.ej. dominios de Lenguaje escrito y Habilidades matemáticas). La Tabla 3 ofrece una breve descripción de cada componente de la BANEDI, así como la edad o el año escolar sugerido para su aplicación. 


\section{Tabla 3}

Componentes de la BANEDI

\begin{tabular}{|c|c|c|}
\hline Componente de la BANEDI & $\begin{array}{l}\text { Edad/año } \\
\text { escolar de } \\
\text { aplicación }\end{array}$ & Breve descripción de la tarea \\
\hline \multicolumn{3}{|c|}{$\begin{array}{c}\text { Cuestionario de detección de síntomas } \\
\text { afectivos y comportamentales }\end{array}$} \\
\hline Versión padres & 6 a 15 años & Respuesta a preguntas sobre observación de conductas con opciones de respuesta "nunca", "a veces", "siempre" \\
\hline Versión docentes & 6 a 15 años & Respuesta a preguntas sobre observación de conductas con opciones de respuesta "nunca", "a veces", "siempre" \\
\hline \multicolumn{3}{|c|}{ Cuestionario de integración sensorial } \\
\hline Versión padres & 6 a 15 años & Respuesta a preguntas sobre observación de conductas con opciones de respuesta "nunca", "a veces", "siempre" \\
\hline Versión docentes & 4 y 5 años & Respuesta a preguntas sobre observación de conductas con opciones de respuesta "nunca", "a veces", "siempre" \\
\hline \multicolumn{3}{|l|}{ Memoria } \\
\hline 1.1 Lista de palabras & 4 a 15 años & Recuerdo inmediato de palabras presentadas oralmente \\
\hline $1.2 \mathrm{El} \mathrm{mapa}$ & 4 a 9 años & Recuerdo inmediato de la ubicación espacial de objetos presentados visualmente \\
\hline $1.3 \mathrm{El}$ circo & 4 a 15 años & Recuerdo inmediato de una historia presentada oralmente \\
\hline 1.4 Lista de palabras II & 4 a 15 años & Recuerdo diferido de palabras presentadas oralmente \\
\hline 1.5 El mapa II & 4 a 9 años & Recuerdo diferido de la ubicación espacial de objetos presentados visualmente \\
\hline $1.6 \mathrm{El}$ circo II & 4 a 15 años & Recuerdo diferido de una historia presentada oralmente \\
\hline $1.7 \mathrm{El}$ topo & 4 a 15 años & Reproducción implícita/inconsciente de una secuencia repetida de estímulos viso-espaciales \\
\hline $1.8 \mathrm{El}$ topo II & 4 a 15 años & Reproducción explícita/consciente de una secuencia repetida de estímulos viso-espacial \\
\hline 1.9 Animales & 4 a 15 años & Reproducción implícita/ inconsciente de una secuencia repetida de estímulos auditivo-verbales \\
\hline 1.10 Animales II & 4 a 15 años & Reproducción explícita/consciente de una secuencia repetida de estímulos auditivo-verbales \\
\hline \multicolumn{3}{|c|}{ Atención y Funciones ejecutivas } \\
\hline 2.1 Draco & 4 a 15 años & Selección de un estímulo visual objetivo entre una serie de distractores \\
\hline $2.2 \mathrm{El} \mathrm{barco}$ & 4 a 15 años & Selección de un estímulo auditivo-verbal objetivo entre una serie de distractores \\
\hline $2.3 \mathrm{El}$ espacio & 4 a 15 años & Respuesta motora a un estímulo visual objetivo por un tiempo prolongado \\
\hline 2.4 Nube & 4 a 15 años & Respuesta motora a un estímulo auditivo-verbal objetivo por un tiempo prolongado \\
\hline 2.5 Números y personas & 4 a 15 años & Recuerdo y secuenciación categórica de palabras presentadas oralmente \\
\hline 2.6 Colores al revés & 4 a 15 años & Recuerdo e inversión de una secuencia de estímulos visuales \\
\hline 2.7 Juegos & 4 a 15 años & Ejecución, coordinación y monitoreo de varias tareas motoras en simultáneo \\
\hline
\end{tabular}




\begin{tabular}{|c|c|c|}
\hline Componente de la BANEDI & $\begin{array}{l}\text { Edad/año } \\
\text { escolar de } \\
\text { aplicación }\end{array}$ & Breve descripción de la tarea \\
\hline \multicolumn{3}{|l|}{ Teoría de la Mente } \\
\hline 3.1 Caras & 4 y 5 años & Nombramiento de emociones a partir de la presentación visual de expresiones faciales \\
\hline 3.2 Sentimientos & 4 y 5 años & Identificación de estados emocionales ajenos a partir de la presentación auditivo-verbal y visual de situaciones \\
\hline 3.3 Historias & 4 y 5 años & Identificación de estados emocionales ajenos a partir de la presentación auditivo-verbal y visual de situaciones \\
\hline 3.4 Historias II & 6 a 8 años & Identificación de estados emocionales ajenos a partir de la presentación auditivo-verbal y visual de situaciones \\
\hline \multicolumn{3}{|l|}{ Habilidades metalingüísticas } \\
\hline 4.1 Unión de sonidos & 6 y 7 años & Unión de fonemas para formar una palabra \\
\hline 4.2 Omisión de sonidos & 6 y 7 años & Formación una nueva palabra omitiendo un sonido \\
\hline 4.3 Conteo de sonidos & 5 a 7 años & Conteo de fonemas contenidos en una palabra \\
\hline 4.4 Separación de sonidos & 6 y 7 años & Identificación de sonidos contenidos en una palabra \\
\hline \multicolumn{3}{|l|}{ Lenguaje oral } \\
\hline 5.1 Palabas iguales y diferentes & 4 años & Identificación de palabras iguales y diferentes \\
\hline 5.2 Movimientos de la boca & 4 años & Imitación de gestos faciales \\
\hline 5.3 Repetición de palabras & 4 años & Repetición de palabras \\
\hline 5.4 Repetición de palabras inventadas & 4 a 8 años & Repetición de pseudopalabras \\
\hline 5.5 Palabras incompletas & 4 a 8 años & Reconocimiento de palabras incompletas \\
\hline $5.6 \mathrm{El}$ comedor & 4 a 6 años & Nombramiento de objetos \\
\hline $5.7 \mathrm{El}$ comedor II & 4 a 6 años & Señalamiento de objetos nombrados \\
\hline 5.8 Comidas, animales y profesiones & 4 a 15 años & Nombramiento rápido de objetos de una categoría semántica \\
\hline 5.9 Picnic & 4 a 8 años & Seguimiento de instrucciones \\
\hline 5.10 Doble sentido & 8 a 15 años & Explicación de frases con doble sentido \\
\hline 5.11 Imágenes y frases & 4 y 5 años & Producción de una frase a partir de una imagen \\
\hline 5.12 Palabras y frases & 6 a 15 años & Producción de una frase a partir de una serie de palabras \\
\hline 5.13 Frases incorrectas & 5 a 8 años & Corrección de frases incorrectas \\
\hline 5.14 Juegos, comidas y ropa & 4 a 8 años & Selección de objetos de una determinada categoría semántica \\
\hline 5.15 Situaciones & 4 a 15 años & Producción de un discurso verbal a partir de presentación de situaciones \\
\hline 5.16 Comprensión del discurso & 4 a 7 años & Selección de imágenes a partir de la presentación de situaciones \\
\hline
\end{tabular}




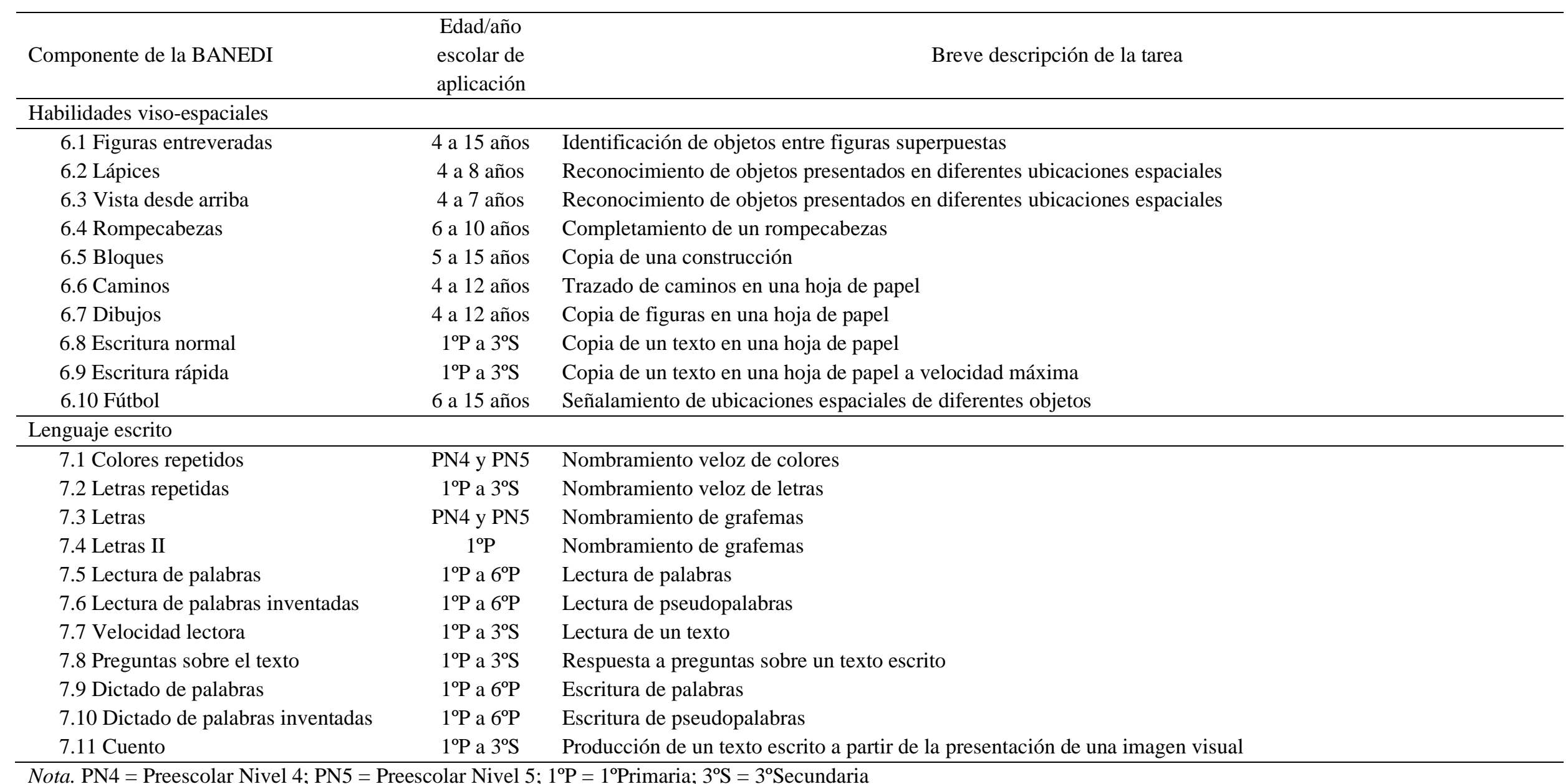

Nota. PN4 $=$ Preescolar Nivel 4 ; PN5 $=$ Preescolar Nivel $5 ; 1^{\circ} \mathrm{P}=1^{\circ}$ Primaria; $3^{\circ} \mathrm{S}=3^{\circ}$ Secundaria 


\begin{tabular}{lcl}
\hline Componente de la BANEDI & $\begin{array}{c}\text { Edad/año } \\
\text { escolar de } \\
\text { aplicación }\end{array}$ & Breve descripción de la tarea \\
\hline Habilidades matemáticas & PN4 & Recitado de la serie numérica directa \\
\hline 8.1 Conteo & PN5 a $3^{\circ} \mathrm{P}$ & Recitado de la serie numérica inversa \\
8.2 Conteo al revés & PN4 a $3^{\circ} \mathrm{S}$ & Comparación de la magnitud de dos conjuntos de puntos \\
8.3 Conjuntos de puntos & PN4 a $3^{\circ} \mathrm{S}$ & Emparejamiento de conjuntos de puntos con palabras número presentadas en forma auditiva \\
8.4 Puntos y palabras-número & PN5 a $3^{\circ} \mathrm{S}$ & Emparejamiento de conjuntos de puntos con número arábigos presentadas en forma visual \\
8.5 Puntos y números arábigos & PN5 a $3^{\circ} \mathrm{S}$ & Comparación de números arábigos presentados visualmente \\
8.6 Números arábigos & $1^{\circ} \mathrm{P} \mathrm{a} 6^{\circ} \mathrm{P}$ & Producción escrita de números arábigos a partir de la presentación auditiva-verbal de una palabra-número \\
8.7 Dictado de números & $1^{\circ} \mathrm{P} \mathrm{a} 6^{\circ} \mathrm{P}$ & Producción oral de palabras-números a partir de la presentación visual de números arábigos \\
8.8 Lectura de números & PN5 a $3^{\circ} \mathrm{S}$ & Ubicación de números arábigos en una recta numérica \\
8.9 Recta numérica & $1^{\circ} \mathrm{P} \mathrm{a} 3^{\circ} \mathrm{S}$ & Identificación de cálculos simples presentados oralmente con palabras-número como correctos o incorrectos \\
8.10 Cuentas con palabras-número & $1^{\circ} \mathrm{P} \mathrm{a} 3^{\circ} \mathrm{S}$ & Identificación de cálculos simples presentados visualmente con números arábigos como correctos o incorrectos \\
8.11 Cuentas con números arábigos & $1^{\circ} \mathrm{P} \mathrm{a} 3^{\circ} \mathrm{S}$ & Resolución de cálculos complejos presentados visualmente con números arábigos \\
8.12 Cuentas escritas & PN5 a $3^{\circ} \mathrm{S}$ & Resolución de problemas matemáticos presentados en formato auditivo-verbal oral y escrito \\
8.13 Problemas matemáticos & &
\end{tabular}




\subsection{Procedimiento}

El presente estudio fue aprobado por la Comisión de Ética de Investigación en Seres Humanos de la Facultad de Medicina de la Universidad Centro Latinoamericano de Economía Humana (CLAEH) del Uruguay el 16 de Noviembre del año 2017. Luego de que los padres de los niños/adolescentes incluidos en la muestra firmaran los consentimientos informados entregados, pedimos que completaran los cuestionarios de detección de síntomas afectivos y comportamentales y de integración sensorial. También solicitamos al docente principal de cada participante completar la versión paralela de estos cuestionarios.

La evaluación se completó en dos sesiones individuales de aprox. 60 a 75 minutos realizadas en un salón tranquilo adjudicado por la institución educativa para este fin. Todos los estímulos fueron presentados en iPads de 10.5" o en protocolos de papel, dependiendo de los requerimientos de cada prueba. La evaluación fue llevada a cabo por profesionales y/o estudiantes avanzados de fonoaudiología, magisterio, psicología, psicopedagogía y psicomotricidad (Montevideo: $n=33$ y Buenos Aires: $n=35$ ). Además de los materiales nombrados anteriormente, cada evaluador contó con una computadora para seguir los pasos del proceso de evaluación en el software de la BANEDI. En la así llamada "pantalla del evaluador" detallamos el orden de aplicación de las pruebas, las instrucciones para cada prueba, así como los criterios de aplicación y corrección. También incluimos un espacio para notas, en el que el evaluador podía registrar todo tipo de observaciones sobre el proceso de evaluación. La fase de recolección de datos fue supervisada por la segunda, tercera y cuarta autora de este reporte.

Previo a la recolección de datos, el equipo de evaluadores participó de una serie de jornadas de capacitación coordinadas por la segunda, tercera y cuarta autora de este reporte. En esta instancia, además de presentar los criterios de aplicación y corrección para cada prueba, realizamos juegos de roles y entregamos guías de corrección con ejemplos de respuestas de niños/adolescentes. También creamos un grupo de Whatsapp de soporte para poder responder de forma inmediata a cualquier consulta que surgiera por parte de los evaluadores durante el proceso de recolección de datos.

\section{Resultados}

Este reporte se centra en el análisis de las propiedades psicométricas de la BANEDI y focaliza en (1) los resultados de los análisis de parametrización de los ítems de las pruebas y (2) la validez de contenido, de criterio y evolutiva de la BANEDI. 
Antes de presentar los resultados de los análisis descritos anteriormente, queremos resaltar que la construcción y validación de un test psicológico nunca representa un proceso completamente finalizado. En cambio, el instrumento debe ser periódicamente revisado y complementado con nuevas evidencias que avalen los distintos usos a los que está dirigido (Fernández-Pinto, Santamaría, Sánchez-Sánchez, Carrasco \& del Barrio, 2015). Este es uno de los principios claves que caracterizan a la BANEDI. Por lo tanto, en muchos casos aún estamos realizando análisis estadísticos complementarios para obtener más información sobre la calidad psicométrica de la BANEDI y realizar ajustes pertinentes. Estos casos serán indicados adecuadamente en la información presentada.

\subsection{Análisis de parametrización de los ítems de cada prueba}

Siguiendo la Teoría Clásica de los Test (TCT), en primer lugar calculamos índices de dificultad $p$ y de discriminación $r$ para los ítems contenidos en cada prueba. De esta forma apuntamos a determinar (a) si los ítems contenidos en cada prueba eran lo suficientemente fáciles y difíciles y (b) si lograban discriminar entre niños/adolescentes con una mejor o peor competencia en la habilidad subyacente que la prueba pretendía medir.

Sin embargo, este tipo de análisis solamente resulta adecuado en el caso de pruebas en las que sea posible distinguir claramente ítems independientes a los que el niño/adolescente debe responder. Por lo contrario, no es pertinente en pruebas que solamente constan de un ítem, como por ejemplo la prueba 8.1 Conteo en el dominio de Habilidades Matemáticas. Tampoco es pertinente para pruebas en las que no sea posible distinguir ítems independientes, como por ejemplo en la prueba 6.5 Bloques en el dominio de Habilidades viso-espaciales. Para superar estas limitaciones para el cálculo de los índices de dificultad y de discriminación dentro del marco de la Teoría Clásica de los Test (TCT), decidimos complementar nuestros análisis por medio del cálculo de índices de dificultad y

discriminación dentro del marco de la Teoría de Respuesta al Ítem y (TRI - p.ej. Hambleton, Swaminathan \& Rogers, 1991).

Todos los análisis de parametrización de las pruebas de la BANEDI fueron realizados en el programa estadístico $R$ (R Core Team, 2013) con los paquetes psychometric (Fletcher, 2010), ltm (Rizopoulos, 2006) y cIRT (Culpepper \& Balamuta, 2015). Los resultados para la mayoría de las pruebas reflejaron que los ítems cubrían niveles de dificultad variados, desde muy fáciles hasta muy difíciles. También se observaron niveles de discriminación adecuados para la mayoría de las pruebas. Para 13 de las 75 pruebas (1.8 El Topo II, 1.10 Animales II, 2.7 Juegos, 3.4 Historias II, 5.8 Comidas, animales y profesiones, 6.5 Bloques, 6.8 Escritura 
normal, 6.9 Escritura rápida, 7.1 Colores repetidos, 7.2 Letras repetidas, 7.7 Velocidad lectora, 8.1 Conteo y 8.2 Conteo al revés) no es pertinente calcular índices de dificultad y discriminación ya que estas pruebas solamente cuentan con un ítem. Finalmente, aún estamos realizando análisis complementarios tomando en cuenta medidas de tiempos de reacción para cada ítem para 21 pruebas (1.3 a 1.7 y 1.9 en el dominio de Memoria, 5.14 en el dominio de Lenguaje oral, 6.1 a 6.4 y 6.6 en el dominio de Habilidades viso-espaciales, 7.5 y 7.11en el dominio de Lenguaje escrito y 8.3 a 8.6 y 8.9 a 8.11 en el dominio de Habilidades matemáticas) con el propósito de evaluar su grado de dificultad y poder discriminatorio. Si bien esta información fue recolectada durante el estudio de tipificación, su extracción del software requiere de pasos adicionales de procesamiento de la información, que a la fecha aún se encuentran en proceso. Una vez que esta información se encuentre disponible, será actualizada de forma inmediata en una nueva versión del manual de la BANEDI disponible en https://osf.io/c6vqp/. En el Anexo A presentamos detalles adicionales sobre los resultados del análisis de parametrización de los ítems de cada una de las 75 pruebas de la BANEDI.

En base a los resultados de estos análisis a la fecha, eliminamos ítems que resultaron muy fáciles (TCT: $p>.75$; TRI: $\beta<-2$ ) o muy difíciles (TCT: $p<.25$; TRI: $\beta>2$ ) para la mayoría de los niños/adolescentes y/o que no lograron discriminar entre niños/adolescentes con diferentes niveles de rendimiento (TCT: $r<.30$, Cohen, 1988; TRI: $\alpha>0.64$, Hambleton et al., 1991). En algunos casos la información obtenida por los análisis de parametrización sirvió para limitar el rango de aplicación de algunas pruebas a edades/años escolares en los cuales los ítems sí mostraron niveles acordes de dificultad y discriminación. Los mismos criterios serán aplicados para los ítems de las pruebas, para las cuales aún estamos realizando análisis complementarios.

\subsection{Análisis de validez}

Evaluamos tres aspectos de la validez de la BANEDI: (1) la validez de contenido, (2) la validez de criterio y (3) la validez evolutiva.

3.2.1 Validez de contenido. Uno de los primeros pasos de la elaboración de la BANEDI consistió en la consulta con un panel de expertos para analizar si el contenido de la BANEDI constituía una muestra representativa de los constructos que pretendíamos medir (Martínez Arias, 1995). Para este fin contactamos a cinco investigadores con conocimientos especializados en la evaluación de al menos uno de los dominios de funcionamiento neuropsicológico de la BANEDI y les presentamos la primera versión de pruebas e ítems construidos antes de la administración piloto y del estudio de tipificación. 
Les pedimos que en una escala tipo Likert de tres niveles $(1$ = nada claro/relevante/coherente/suficiente, 2 = algo claro/relevante/coherente/suficiente y 3 = muy claro/relevante/coherente/suficiente) juzgaran la claridad, relevancia, coherencia y suficiencia de los ítems contendidos en cada prueba de la BANEDI. La categoría de claridad fue definida como la fácil comprensión de las consignas de las pruebas, así como de los ítems individuales incluidos en cada prueba. La categoría de relevancia apuntó a medir si el ítem o la prueba resultaba esencial, mientras la categoría de coherencia refirió a la pertenencia del ítem a la prueba en la que estaba incluida. Finalmente, la categoría de suficiencia se centró en juzgar si los ítems pertenecientes a cada prueba bastaban para obtener una medición exacta de los subcomponentes neuropsicológicos que se pretendían evaluar. En la Tabla 4, presentamos un resumen del promedio de juicios emitidos para cada dominio de funcionamiento neuropsicológico de la BANEDI.

\section{Tabla 4}

Promedio de juicios de expertos

\begin{tabular}{lcccc}
\hline \multicolumn{1}{c}{ Dominio } & Claridad & Relevancia & Coherencia & Suficiencia \\
\hline Habilidades metalingüísticas & 2.75 & 3.00 & 3.00 & 3.00 \\
Lenguaje oral & 2.88 & 2.88 & 2.71 & 2.71 \\
Habilidades viso-espaciales & 2.90 & 3.00 & 3.00 & 3.00 \\
Lenguaje escrito & 3.00 & 2.73 & 3.00 & 2.36 \\
Habilidades matemáticas & 3.00 & 2.69 & 2.85 & 2.85 \\
\hline
\end{tabular}

A grandes rasgos los juicios de expertos confirmaron la validez de contenido de la BANEDI mostrando que los ítems y las pruebas que la componen eran lo suficientemente claros, relevantes, coherentes y suficientes para medir el desarrollo neuropsicológico de niños/adolescentes. Con el fin de mejorar la calidad de la BANEDI incorporamos todas las sugerencias puntuales que los expertos ofrecieron en relación a ítems y pruebas determinadas antes de proceder al estudio piloto del instrumento y finalmente al estudio de tipificación.

3.2.2 Validez de criterio. Examinamos hasta qué punto los resultados de las pruebas de la BANEDI estaban asociados con tres fuentes de información sobre el mismo constructo subyacente, que pretendíamos medir: (1) juicios docentes sobre las habilidades de los niños/adolescentes que participaron de la tipificación de la BANEDI, (2) puntajes alcanzados en otros test empleados en el medio y (3) diagnósticos clínicos de trastornos neuropsicológicos. 
3.2.2.1 Validez concurrente con juicios docentes. Para evaluar si los puntajes alcanzados por los niños/adolescentes que participaron de la tipificación de la BANEDI estaban asociados con las observaciones de sus docentes, solicitamos juicios docentes sobre las habilidades de lenguaje oral, gráficas, de lectura y de escritura para un subgrupo de niños/adolescentes de la muestra total $(N=45)$ en base a las siguientes tres categorías: (a) sin dificultad, (b) dificultad leve y (c) gran dificultad. Con esta información calculamos correlaciones de Pearson $r$ entre los juicios docentes y algunas pruebas de la BANEDI, que pretendían evaluar constructos similares a los observados por los docentes en el aula. La Tabla 5 muestra los resultados de dichos análisis.

\section{Tabla 5}

Correlación de Pearson entre juicio docente y pruebas de Lenguaje oral, Habilidades visoespaciales y Lenguaje escrito, respectivamente.

\begin{tabular}{lll}
\hline Prueba & $N$ & $r$ \\
\hline Lenguaje oral & & \\
5.4 Repetición de palabras inventadas & 32 & $.74 * * *$ \\
5.5 Palabras incompletas & 32 & $.50 * *$ \\
5.6 El comedor & 20 & .07 \\
5.7 El comedor II & 20 & .20 \\
5.8 Comidas, animales y profesiones & 45 & .06 \\
5.9 Picnic & 32 & .24 \\
5.10 Doble sentido & 19 & .15 \\
5.11 Imágenes y frases & 16 & .01 \\
5.12 Palabras y frases & 29 & .25 \\
5.13 Frases incorrectas & 32 & $.64 * * *$ \\
5.14 Juegos, comidas y ropa & 31 & .15 \\
5.15 Situaciones & 45 & $.30 *$ \\
5.16 Comprensión del discurso & 26 & $.55 * *$ \\
Habilidades viso-espaciales & & \\
6.6 Caminos & 18 & .25 \\
6.7 Dibujos & 18 & $.57 *$ \\
Lenguaje escrito & & \\
7.5 Lectura de palabrasa & 27 & $.44 *$ \\
7.6 Lectura de palabras inventadasa & 27 & .26 \\
7.7 Velocidad lectora & 27 & $.40 *$ \\
7.8 Preguntas sobre el texto & 27 & .11 \\
7.9 Dictado de palabras & 27 & .11 \\
7.10 Dictado de palabras inventadas & 27 & .12 \\
7.11 Cuento & 27 & .07 \\
\hline
\end{tabular}

En el dominio de Lenguaje oral encontramos correlaciones significativas entre los juicios docentes y los puntajes alcanzados en las pruebas 5.4 Repetición de palabras inventadas, 5.5 Palabras incompletas, 5.13 Frases incorrectas, 5.15 Situaciones y 5.16 
Comprensión del discurso. Esta evidencia indica que estas pruebas de la BANEDI evalúan constructos similares a los evaluados por docentes en el aula a la hora de medir las habilidades de lenguaje de sus alumnos.

En el dominio de Habilidades viso-espaciales, la prueba 6.7 Dibujos correlacionó significativamente con los juicios docentes sobre las actividades gráficas de sus alumnos y de esta forma confirmó que esta prueba mide habilidades similares a las evaluadas por los docentes de aula en relación a las actividades gráficas de sus alumnos (p.ej. en tareas de geometría).

En el dominio de Lenguaje escrito los resultados reflejaron correlaciones significativas entre las pruebas 7.5 Lectura de palabras y 7.7 Velocidad lectora con los juicios docentes sobre las habilidades de lectura de sus alumnos. En cambio, no encontramos correlaciones significativas con los juicios docentes sobre habilidades de escritura y las pruebas 7.9 Dictado de palabras, 7.10 Dictado de palabras inventadas y 7.11 Cuento. Es posible que pedirle a los docentes que formulen un juicio global sobre las habilidades de escritura de sus alumnos no haya sido suficientemente detallado para captar asociaciones con las pruebas de la BANEDI. Por lo tanto, en futuras revisiones planificamos incluir juicios más detallados, por ejemplo, en relación a las habilidades de ortografía y producción escrita.

3.2.2.2 Validez concurrente con otros instrumentos de evaluación. En forma similar al análisis de juicios docentes, también evaluamos el grado de asociación de los puntajes alcanzados en la BANEDI con otros instrumentos de evaluación empleados en el medio, que pretenden medir constructos subyacentes similares. Así un subgrupo de siete niños/adolecentes de la muestra total, además de completar todas las pruebas de la BANEDI, también participaron de la aplicación del Test de Eficacia Lectora (TECLE - Cuadro, Costa, Trías y Ponce de León, 2009), del Test de Eficacia Ortográfica (TEO - Cuadro, Palombo, Costa \& von Hagen, 2014) y del Test de Eficacia de Cálculo Aritmético (TECA - Singer, Cuadro, Costa \& von Hagen, 2014). Nuevamente calculamos correlaciones de Pearson $r$ entre los puntajes de estos test y determinadas pruebas de la BANEDI, que pretenden evaluar constructos subyacentes similares. La Tabla 6 muestra los resultados de dichos análisis. 


\section{Tabla 6}

Correlación de Pearson entre TECLE, TEO y TECA y pruebas de la BANEDI

\begin{tabular}{llllll}
\hline Prueba de BANEDI & rTECLE & rTEO & rTECA.Suma & rTECA.Resta & rTECA.Multiplicación \\
\hline Lenguaje escrito & & & & & \\
7.5 Lectura de palabrasa & $.82 *$ & .68 & .58 & .35 & .75 \\
7.7 Velocidad lectora & $.84 *$ & .75 & .38 & .18 & .57 \\
7.9 Dictado de palabras & .69 & $.80 *$ & .34 & .09 & .64 \\
Habilidades matemáticas & & & & & \\
8.10 Cuentas con palabras-número & .74 & $.78 *$ & .25 & .44 & .38 \\
8.10 Cuentas con palabras-númeroa & .16 & .08 & .11 & .09 & .08 \\
8.11 Cuentas con números arábigos & .14 & .14 & .74 & .57 & .25 \\
8.11 Cuentas con números arábigosa & .21 & .34 & .71 & .52 & .02 \\
\hline
\end{tabular}

Nota. *p $<.05$ (bilateral); $a$ Cálculos en base a tiempos de reacción.

En el dominio de Lenguaje escrito los puntajes de la prueba 7.5 Lectura de palabras y 7.7 Velocidad lectora mostraron correlaciones significativas con los puntajes del TECLE. En forma similar encontramos una correlación significativa entre los puntajes de la prueba 7.9 Dictado de palabras y el TEO. Estos resultados indican que las pruebas de la BANEDI nombradas anteriormente evalúan constructos similares a los test TECLE y TEO, comúnmente empleados en nuestro medio.

En cambio, en el dominio de Habilidades Matemáticas las pruebas 8.10 Cuentas con palabras-número y 8.11 Cuentas con números arábigos no mostraron correlaciones significativas con el TECA. Si bien tanto el TECA, como estas pruebas de la BANEDI pretenden evaluar la precisión y velocidad para realizar cálculos aritméticos, también existen varias diferencias entre estos instrumentos, que podrían explicar los resultados presentados en la Tabla 6. Así en la BANEDI los cálculos son presentados en formato digital en la pantalla, mientras el TECA es un test de lápiz y papel. Además, en las pruebas de la BANEDI el niño/adolescente debe responder si el cálculo presentado es correcto o incorrecto, mientras en el TECA la tarea consiste en seleccionar la respuesta correcta entre cuatro opciones. Es posible, que estas diferencias, entre otras, exijan el involucramiento de diferentes subprocesos cognitivos y de esta forma expliquen por qué no encontramos correlación entre los puntajes obtenidos en ambos test.

\subsubsection{Validez concurrente con diagnósticos clínicos de trastornos}

neuropsicológicos. Para investigar la validez concurrente de la BANEDI con diagnósticos independientes de trastornos neuropsicológicos, analizamos el rendimiento de once casos clínicos con un diagnóstico previo de trastornos específicos del lenguaje en las pruebas del dominio de Habilidades metalingüísticas, Lenguaje oral y Lenguaje escrito de la BANEDI. 
Los niños/adolescentes habían sido diagnosticados formalmente por un fonoaudiólogo en años anteriores a la aplicación de la BANEDI. Los informes diagnósticos evidenciaron una gran variedad de subtipos de trastornos específicos del lenguaje. Mientras los déficit de algunos niños/adolescentes solo se concentraban en las habilidades de lenguaje expresivo (p.ej. vocabulario expresivo, producción de frases), en otros también las habilidades de lenguaje receptivo (p.ej. comprensión de indicaciones) estaban alteradas. En la Tabla 7 presentamos los datos demográficos de los niños/adolescentes evaluados.

\section{Tabla 7}

Datos demográficos de los niños/adolescentes con trastornos específicos del lenguaje evaluados con la BANEDI

\begin{tabular}{llll}
\hline Caso clínico & Sexo & Edad & Año escolar \\
\hline 1 & $\mathrm{M}$ & 6 & Preescolar Nivel 5 \\
2 & $\mathrm{M}$ & 6 & $1^{\circ}$ Primaria \\
3 & $\mathrm{M}$ & 7 & $1^{\circ}$ Primaria \\
4 & $\mathrm{M}$ & 8 & $2^{\circ}$ Primaria \\
5 & $\mathrm{~F}$ & 8 & $2^{\circ}$ Primaria \\
6 & $\mathrm{M}$ & 8 & $3^{\circ}$ Primaria \\
7 & $\mathrm{~F}$ & 9 & $3^{\circ}$ Primaria \\
8 & $\mathrm{M}$ & 12 & $6^{\circ}$ Primaria \\
9 & $\mathrm{~F}$ & 12 & $6^{\circ}$ Primaria \\
10 & $\mathrm{~F}$ & 13 & $1^{\circ}$ Secundaria \\
11 & $\mathrm{M}$ & 13 & $1^{\circ}$ Secundaria \\
\hline
\end{tabular}

Anticipamos que si la BANEDI es una herramienta válida para detectar niños/adolescentes con este tipo de trastornos neuropsicológicos, los once niños/adolescentes evaluados debían mostrar puntajes dentro de la categoría diagnóstica "muy descendido" (puntaje $z<-2.00$ o percentil $<5$ ) en por lo menos una prueba del dominio de Habilidades metalingüísticas, Lenguaje oral y Lenguaje escrito. Para comprobar esta hipótesis, seguimos los procedimientos estadísticos propuestos por Crawford y Howell (1998) y Crawford, Garthwaite y Porter (2010). Estos autores desarrollaron un programa informático llamado SINGLIMS para identificar si el puntaje alcanzado por un solo individuo es significativamente diferente del puntaje obtenido por la media de un grupo control. La metodología de Crawford y Howell (1998) y Crawford et al. (2010) ha sido empleada ampliamente para estudiar casos clínicos tanto en el área de trastornos neuropsicológicos adquiridos, como en trastornos del desarrollo neuropsicológico (Colenbrander, Kohnen, Smith-Lock \& Nickels, 2016; Couto et al., 2013; Dalrymple, Elison \& Duchaine, 2017; Friedmann \& Novogrodsky, 2011; Kambanaros, Michaelides \& Grohmann, 2015; Novogrodsky \& Friedmann, 2006; Peyrin et al., 2012). En la Tabla 8 y 9 presentamos los 
resultados de dicho análisis en forma de valores $t$. Las celdas marcadas en gris representan los valores, que indican una diferencia estadísticamente significativa entre el puntaje alcanzado por el niño/adolescente y el grupo control. 


\section{Tabla 8}

Resultados de análisis SINGLIMS según Crawford y Howell (1998) y Crawford et al. (2010) para pruebas de Habilidades metalingüísticas y Lenguaje oral

\begin{tabular}{|c|c|c|c|c|c|c|c|c|c|c|c|}
\hline \multirow{2}{*}{ Prueba } & \multicolumn{11}{|c|}{ Caso clínico } \\
\hline & 1 & 2 & 3 & 4 & 5 & 6 & 7 & 8 & 9 & 10 & 11 \\
\hline 4.1 Unión de sonidos & -1.31 & -1.01 & -1.72 & NA & NA & NA & NA & NA & NA & NA & NA \\
\hline 4.2 Omisión de sonidos & -1.31 & -1.01 & -1.72 & NA & NA & NA & NA & NA & NA & NA & NA \\
\hline 4.3 Conteo de sonidos & -1.31 & -1.21 & -1.50 & NA & NA & NA & NA & NA & NA & NA & NA \\
\hline 4.4 Separación de sonidos & -1.76 & -1.76 & -1.76 & NA & NA & NA & NA & NA & NA & NA & NA \\
\hline 5.2 Movimientos de la bocaa & -0.49 & -1.00 & 1.04 & NA & NA & NA & NA & NA & NA & NA & NA \\
\hline 5.3 Repetición de palabrasa & -1.78 & -0.18 & $-4.46 * * *$ & 0.35 & -0.18 & 0.88 & NA & NA & NA & NA & NA \\
\hline 5.4 Repetición de palabras inventadas & $-3.73^{* * *}$ & -1.13 & $-6.38^{* * *}$ & -0.78 & -0.78 & -0.78 & NA & NA & NA & NA & NA \\
\hline 5.5 Palabras incompletas & -1.14 & $-2.24 *$ & $-4.40 * * *$ & -0.40 & $-2.60 * *$ & -1.13 & NA & NA & NA & NA & NA \\
\hline $5.6 \mathrm{El} \mathrm{comedorb}$ & $-2.47 *$ & -0.23 & $-2.47 *$ & -0.23 & -0.23 & -0.23 & NA & NA & NA & NA & NA \\
\hline 5.7 El comedor $\mathrm{IIb}_{\mathrm{b}}$ & $-2.04 *$ & 0.68 & $-2.04 *$ & -0.68 & -0.68 & 0.68 & NA & NA & NA & NA & NA \\
\hline 5.8 Comidas, animales y profesiones & -0.08 & -0.56 & $-3.55 * * *$ & -1.37 & $-2.43 *$ & $-2.12 *$ & -0.55 & -0.21 & -0.72 & $-2.03 *$ & -0.15 \\
\hline 5.9 Picnic $_{\mathrm{c}}$ & $-3.46 * * *$ & -1.38 & -0.61 & $-4.51 * * *$ & $-2.34 *$ & -0.89 & -0.89 & -0.17 & 0.18 & -1.62 & -0.17 \\
\hline 5.10 Doble sentido & NA & NA & NA & -1.42 & -1.42 & -1.42 & 0.95 & -0.45 & 0.59 & $-3.72 * * *$ & $-3.33 * *$ \\
\hline 5.12 Palabras y frases & -1.64 & -0.90 & $-3.50 * * *$ & -0.48 & $-3.39 * *$ & $-2.22 *$ & -0.91 & -0.05 & 0.76 & $-3.40 * *$ & -1.68 \\
\hline 5.13 Frases incorrectasc & $-2.83 * *$ & $-2.83^{* *}$ & $-4.73 * * *$ & $-4.81 * * *$ & $-4.81 * * *$ & $-6.62^{* * *}$ & -1.19 & NA & NA & NA & NA \\
\hline 5.14 Juegos. comidas y ropa & -0.69 & -0.05 & -0.11 & $-8.77 * * *$ & 0.04 & -0.90 & NA & NA & NA & NA & NA \\
\hline 5.15 Situaciones & $-3.68^{* * *}$ & -0.84 & -1.14 & -0.56 & 0.63 & 0.63 & NA & 0.44 & 0.44 & -0.86 & -0.86 \\
\hline 5.16 Comprensión del discursod & -0.17 & 1.05 & -0.66 & $-2.15 *$ & $-2.15 *$ & -0.66 & NA & NA & NA & NA & NA \\
\hline
\end{tabular}

Nota. ${ }^{* * *} p<.001$ (bilateral); $* p<.01$ (bilateral); $* p<.05$ (bilateral); NA = No aplica; aComparación con datos empíricos de niños de 4 años; bComparación con datos

empíricos de niños de 6 años; cComparación con datos empíricos de niños de 6, 7 y 8 años; dComparación con datos empíricos de niños de 7 y 8 años; Las celdas marcadas en gris indican una diferencia significativa entre el puntaje del niño/adolescente y el puntaje medio alcanzado por niños/adolescentes de la misma edad/año escolar. 


\section{Tabla 9}

Resultados de análisis SINGLIMS según Crawford y Howell (1998) y Crawford et al. (2010) para pruebas de Lenguaje escrito

\begin{tabular}{|c|c|c|c|c|c|c|c|c|c|c|c|}
\hline \multirow{2}{*}{ Prueba } & \multicolumn{11}{|c|}{ Caso clínico } \\
\hline & 1 & 2 & 3 & 4 & 5 & 6 & 7 & 8 & 9 & 10 & 11 \\
\hline 7.2 Letras repetidas & 0.10 & 0.10 & -0.62 & $-2.30 *$ & 0.32 & 0.32 & 0.10 & $-3.90 * * *$ & $-3.90 * * *$ & 0.32 & -0.27 \\
\hline 7.2 Letras repetidasa & $-5.69 * * *$ & $-3.61 * * *$ & -1.09 & -1.02 & $-2.52 *$ & -0.64 & -0.24 & $-2.87 * *$ & -1.51 & -1.39 & 0.11 \\
\hline 7.3 Letrasb & 1.01 & 1.01 & NA & NA & NA & NA & NA & NA & NA & NA & NA \\
\hline 7.4 Letras $\mathrm{II}_{\mathrm{c}}$ & NA & -1.71 & -1.71 & 0.02 & -1.01 & NA & NA & NA & NA & NA & NA \\
\hline 7.5 Lectura de palabras & NA & NA & $-3.42 * * *$ & -0.42 & 0.28 & $-2.05 *$ & $-3.36 * *$ & $-3.49 * * *$ & 0.32 & NA & NA \\
\hline 7.5 Lectura de palabrasa & NA & NA & 1.15 & -1.37 & $-2.17 *$ & -1.56 & $-3.52 * * *$ & -1.12 & -1.63 & NA & NA \\
\hline 7.6 Lectura de palabras inventadas & NA & NA & $-2.05 *$ & -0.92 & -0.69 & -0.47 & $-3.36 * *$ & -1.04 & -0.62 & NA & NA \\
\hline 7.6 Lectura de palabras inventadasa & NA & NA & 1.76 & -0.79 & -0.40 & -0.96 & -1.00 & 0.47 & -1.08 & NA & NA \\
\hline 7.7 Velocidad lectora & NA & NA & 0.30 & -0.83 & 0.24 & -1.69 & -1.76 & $-2.19 *$ & -0.25 & -1.96 & -0.77 \\
\hline 7.8 Preguntas sobre el texto & NA & NA & $-2.39 *$ & -0.90 & -0.90 & -0.80 & -0.80 & -1.58 & -0.16 & $-3.35^{* * *}$ & -1.15 \\
\hline 7.9 Dictado de palabras & NA & NA & -1.23 & -1.68 & $-2.13 *$ & -1.00 & -1.00 & $-2.05 *$ & -0.76 & NA & 0.52 \\
\hline 7.10 Dictado de palabras inventadas & NA & NA & $-2.05 *$ & $-2.19 *$ & $-2.19 *$ & -1.64 & -1.26 & -0.75 & -0.21 & NA & NA \\
\hline 7.11 Cuento & NA & NA & $-2.71 * *$ & 0.02 & $-2.27 *$ & -0.28 & 0.30 & NA & -1.05 & NA & NA \\
\hline
\end{tabular}

Nota. $* * * p<.001$ (bilateral); $* *<.01$ (bilateral); $* p<.05$ (bilateral); NA = No aplica; aCálculos basados en tiempos de reacción; bComparación con datos empíricos de niños de 5 años; cComparación con datos empíricos de niños de $1^{\circ}$ Primaria. Las celdas marcadas en gris indican una diferencia significativa entre el puntaje del niño/adolescente y el puntaje medio alcanzado por niños/adolescentes de la misma edad/año escolar 
Los resultados muestran que los once casos clínicos de niños/adolescentes con diagnósticos previos de trastornos específicos del lenguaje rindieron significativamente por debajo del grupo control en al menos una de las pruebas de los dominios de Habilidades metalingüísticas, Lenguaje oral y Lenguaje escrito de la BANEDI. Estos datos subrayan la validez de la BANEDI para detectar trastornos neuropsicológicos del desarrollo. En futuras revisiones apuntaremos a ampliar esta evidencia para otros tipos de trastornos neuropsicológicos del desarrollo (p.ej. discalculia, trastorno por déficit atencional, etc.).

\subsubsection{Validez evolutiva}

Específicamente en el caso de test que evalúan constructos subyacentes que siguen patrones evolutivos, una forma de determinar la validez del instrumento consiste en analizar los puntajes obtenidos por diferentes grupos etarios. Así en el caso de test de desarrollo, como la BANEDI, se espera observar un incremento en los puntajes alcanzados a medida que la edad de los niños/adolescentes, que completaron la BANEDI aumenta (Anastasi \& Urbina, 1998). En forma similar se espera que los tiempos de reacción vayan disminuyendo a lo largo del desarrollo.

Para evaluar este aspecto, investigamos el impacto de la variable edad sobre los puntajes directos y tiempos de reacción alcanzados en cada prueba de la BANEDI por medio de análisis de varianza (ANOVA). Para algunas pruebas estos análisis no eran pertinentes, ya que la edad de aplicación de la prueba se limitaba a un solo grupo etario (p.ej. 5.1 Palabras iguales y diferentes del dominio de Lenguaje oral) o porque los ítems presentados a cada grupo etario fueron diferentes (p.ej. 8.12 Cuentas escritas del dominio de Habilidades matemáticas). Para todas las demás pruebas, los resultados exactos se presentan en el Anexo B.

Los resultados reflejaron que para todas las pruebas de la BANEDI se observó un aumento en el puntaje y un descenso en los tiempos de reacción alcanzados en función de la edad de los niños/adolescentes que participaron en la tipificación de la BANEDI, con la excepción de cuatro pruebas (1.7 El Topo, 2.2 El barco, 5.11 Imágenes y frases, 7.8 Preguntas sobre el texto). En primer lugar, en la prueba 1.7 El Topo, que mide la capacidad de aprendizaje implícito de estímulos viso-espaciales, los niños de 4 y 5 años alcanzaron puntajes más altos que los niños/adolescentes de edades más avanzadas. Existen dos posibles interpretaciones para este resultado. Por un lado, es posible, que los niños de 4 y 5 años hayan alcanzado puntajes más altos por dar respuestas aleatorias con un estilo de trabajo impulsivo. Como el tiempo de reacción juega un rol central para calcular el puntaje de esta 
prueba, este tipo de conducta podría explicar por qué no se observa un patrón evolutivo en esta prueba.

Por otro lado, es posible que los niños de 4 y 5 años cuenten con una mayor capacidad de aprendizaje implícito de estímulos viso-espaciales que los niños/adolescentes de mayor edad. Como explicitamos en mayor detalle en el marco teórico, que sustenta la inclusión de esta prueba en la BANEDI (ver dominio de Memoria en el manual de la BANEDI), muchos de los aprendizajes logrados en edades tempranas (p.ej. marcha, expresión de primeras palabras, etc.) se basan en mecanismos implícitos. Recién en edades más avanzadas la capacidad de aprendizaje explícito se desarrolla y juega un rol central para beneficiarse de la instrucción explícita recibida en contextos de educación formal. A futuro profundizaremos los análisis de los resultados de esta prueba para dilucidar cuál de las dos hipótesis descritas anteriormente es sustentada por la evidencia recolectada en el estudio de tipificación de la BANEDI.

En segundo lugar, en la prueba 2.2 El barco los niños de 4 y 5 años mostraron tiempos de reacción más eficaces que sus pares de edades mayores. A su vez, observamos un enlentecimiento de la velocidad de respuesta en los adolescentes de 15 años, en comparación con sus pares de edades menores. Actualmente estamos en proceso de análisis para detectar la causa de este resultado inesperado para poder ajustar esta prueba en una próxima revisión de la BANEDI. La tercera excepción se refiere a la prueba 5.11 Imágenes y frases. Si bien identificamos un aumento en el puntaje medio alcanzado por los niños de 5 años en comparación con los niños de 4 años, este incremento no alcanzó valores estadísticamente significativos. Finalmente, en la prueba 7.8 Preguntas sobre el texto los niños/adolescentes de $6^{\circ}$ de Primaria a $3^{\circ}$ de Secundaria mostraron puntajes medios similares. En base a este hallazgo planificamos incorporar nuevos estímulos con mayores grados de dificultad para estos años escolares en una próxima revisión de la BANEDI.

\section{Discusión}

El presente reporte apunta a describir el proceso de construcción y la evidencia de validez de la BANEDI, un instrumento digital de evaluación del desarrollo neuropsicológico dirigido a niños y adolescentes de 4 a 15 años de la región del Río de la Plata (Montevideo, Uruguay y Buenos Aires, Argentina). Aunque el ajuste de la calidad psicométrica de un instrumento de evaluación es un proceso continuo de constante revisión, los resultados de los análisis presentados en el apartado anterior evidencian que la BANEDI cumple con los criterios de calidad psicométrica necesarios para realizar una estimación válida de las 
funciones neuropsicológicas de niños/adolescentes entre 4 y 15 años de edad y cursando Educación Preescolar Nivel 4 a $3^{\circ}$ de Educación Secundaria en la región del Río de la Plata. Con el fin de continuar fortaleciendo la calidad psicométrica de la BANEDI, seguiremos complementando, profundizando y ampliando los análisis realizados en constantes actualizaciones y revisiones de la BANEDI y reportaremos esta información en nuevas versiones del manual teórico y de aplicación disponible en https:/osf.io/c6vqp/. A continuación discutimos la evidencia presentada en el apartado anterior en relación (1) al grado de dificultad y el poder discriminatorio de los ítems contenidos en las pruebas de la BANEDI y (2) la validez de los componentes del instrumento.

En primer lugar, los ítems de la mayoría de las pruebas de la BANEDI manifiestan índices de dificultad variados, así como índices de discriminación aceptables. Los análisis de parametrización para 21 de las 75 pruebas de la BANEDI aún están siendo procesados, ya que apuntamos a tomar en cuenta los tiempos de reacción empleados para completar cada ítem con el fin de lograr estimaciones más sensitivas. Esta información fue recolectada durante el estudio de tipificación y requiere de pasos adicionales de procesamiento de la información por parte del equipo informático de la BANEDI para extraer los datos del software. Una vez que esta información se encuentre disponible, completaremos los análisis y actualizaremos la información en una nueva versión del manual de la BANEDI disponible en https://osf.io/c6vqp/.

En segundo lugar, los resultados de los análisis psicométricos reflejaron evidencia sobre la validez de la BANEDI confirmando que las puntuaciones obtenidas a partir de su aplicación miden el constructo subyacente que se pretende medir. Por un lado, los juicios de un panel de expertos muestran que los ítems de las pruebas de la BANEDI alcanzan criterios adecuados de claridad, relevancia, coherencia y suficiencia. Por otro lado, las puntuaciones obtenidas por un subgrupo de niños/adolescentes de la muestra de tipificación correlacionan significativamente con los juicios de sus docentes sobre sus habilidades de lenguaje oral, gráficas y de lectura. Como no encontramos una asociación significativa entre los juicios docentes sobre las habilidades de escritura de sus alumnos y las puntuaciones de las pruebas de la BANEDI que pretenden medir dichas habilidades, nos proponemos continuar investigando este aspecto en futuras revisiones del instrumento.

También encontramos evidencia de la validez de la BANEDI en forma de correlaciones significativas entre las puntuaciones de las pruebas del dominio de Lenguaje escrito y el Test de Eficacia Lectora (TECLE - Cuadro et al., 2009) y el Test de Eficacia Ortográfica (TEO - Cuadro et al., 2014) que pretenden evaluar constructos subyacentes 
similares. Sin embargo, no encontramos una asociación significativa entre las puntuaciones de las pruebas del dominio de Habilidades matemáticas y el Test de Eficacia de Cálculo Aritmético (TECA - Singer et al., 2014). Este resultado posiblemente se deba a diferencias en la presentación de los estímulos y la modalidad de respuesta entre ambos instrumentos y será objeto de futuras investigaciones en el marco de la revisión continua de la BANEDI. En forma similar, en el futuro apuntamos a ampliar el estudio de la validez concurrente de la BANEDI con otros instrumentos empleados en el medio (p.ej. LEE. Test de lectura y escritura en español - Defior et al., 2006 y Test de atención d2, Brickenkamp, 2002).

Un resultado especialmente relevante que evidencia la validez de la BANEDI para identificar alteraciones en el desarrollo neuropsicológico, son los puntajes significativamente descendidos de once casos clínicos de niños/adolescentes con diagnósticos previos de trastornos específicos en el lenguaje en la pruebas de los dominio de Lenguaje oral y escrito de la BANEDI. A futuro nos proponemos ampliar estos datos a otras poblaciones de niños/adolescentes con diagnósticos previos de otros subtipos de trastornos del desarrollo (p.ej. trastorno por déficit de atención, discalculia, etc.). Finalmente, los resultados de los análisis de la validez evolutiva de la BANEDI indican que casi todas las pruebas son sensibles para captar patrones evolutivos en el desarrollo neuropsicológico de niños/adolescentes (aumento de puntuaciones y descenso de tiempos de reacción). Solamente en el caso de cuatro pruebas (1.7 El Topo, 2.2 El barco, 5.11 Imágenes y frases, 7.8 Preguntas sobre el texto) es preciso afinar los estímulos en futuras revisiones del instrumento para cumplir con este criterio a lo largo de todos los grupos etarios a los que se dirige la BANEDI.

\section{Conclusión}

En suma, la descripción de la propiedades psicométricas de la BANEDI presentada en este reporte refleja que el instrumento cumple con los criterios de calidad psicométrica necesarios para realizar una estimación válida de las funciones neuropsicológicas de niños/adolescentes entre 4 y 15 años de edad y cursando Educación Preescolar Nivel 4 a $3^{\circ}$ de Educación Secundaria en la región del Río de la Plata. Con el fin de continuar fortaleciendo la calidad psicométrica de la BANEDI, seguiremos complementando, profundizando y ampliando los análisis realizados en constantes actualizaciones y revisiones de la BANEDI. Además, a futuro, dentro de los planes de ampliación de la BANEDI, nos proponemos desarrollar adaptaciones del instrumento y realizar estudios de tipificación que nos permitan contar con baremos individualizados también para otras regiones dentro de Latinoamérica. De esta forma, apuntamos a que la BANEDI contribuya al crecimiento de la neuropsicología 
del desarrollo en Latinoamérica y responda a la necesidad de contar con un instrumento de evaluación neuropsicológica adaptado a la población local y con datos normativos para la región. 


\section{Referencias}

Arango-Lasprilla, J. C., Stevens, L., Morlett Paredes, A., Ardila, A., \& Rivera, D. (2017). Profession of neuropsychology in Latin America. Applied Neuropsychology: Adult, 24(4), 318-330.

Calvo, J. et al. (2013). Las Necesidades Básicas Insatisfechas a partir de los Censos 2011. Montevideo: Ed. Trilce.

Colenbrander, D., Kohnen, S., Smith-Lock, K., \& Nickels, L. (2016). Individual differences in the vocabulary skills of children with poor reading comprehension. Learning and Individual Differences, 50, 210-220.

Couto, B., Sedeno, L., Sposato, L. A., Sigman, M., Riccio, P. M., Salles, A., ... \& Ibanez, A. (2013). Insular networks for emotional processing and social cognition: comparison of two case reports with either cortical or subcortical involvement. cortex, 49(5), $1420-1434$.

Crawford, J. R., \& Howell, D. C. (1998). Regression equations in clinical neuropsychology: An evaluation of statistical methods for comparing predicted and obtained scores. Journal of Clinical and Experimental Neuropsychology, 20(5), 755-762.

Crawford, J. R., Garthwaite, P. H., \& Porter, S. (2010). Point and interval estimates of effect sizes for the case-controls design in neuropsychology: rationale, methods, implementations, and proposed reporting standards. Cognitive neuropsychology, 27(3), 245-260.

Cuadro, A., Costa, D., Trías, D., \& Ponce de León, P. (2009). Evaluación del nivel lector. Test de eficacia lectora (TECLE) de J. Marín y M. Carrillo. Manual Técnico. Montevideo: Prensa Médica.

Cuadro, A., Palombo, A., Costa, D. \& von Hagen, A. (2014). Evaluación de la eficacia ortográfica: Manual Técnico del Test de Eficacia Ortográfica (TEO). Montevideo: Grupo Magro Ediciones.

Cuetos, F., Rodríguez, B., Ruano, E., \& Arribas D (2014). PROLEC-R. Batería de los procesos lectores -revisada. Madrid: TEA Ediciones.

Culpepper, S. A. \&Balamuta, J. J. (2015). cIRT: Choice Item Response Theory. Disponible en https://cran.r-project.org/package=cIRT.

Dalrymple, K. A., Elison, J. T., \& Duchaine, B. (2017). Face-specific and domain-general visual processing deficits in children with developmental prosopagnosia. The Quarterly Journal of Experimental Psychology, 70(2), 259-275. 
Dansilio, S. (2009). La neuropsicología en Uruguay. Revista Neuropsicología, Neuropsiquiatría y Neurociencias, 9(2), 105-112.

Defior et al. (2006). LEE. Test de lectura y escritura en español. Buenos Aires: Paidós.

Dirección Nacional de Relaciones Económicas con las Provincias (DINREP) (2014).

Necesidades Báscias Insatisfechas (NBI). Información censal del año 2010.

Disponible en

http://www2.mecon.gov.ar/hacienda/dinrep/Informes/archivos/NBIAmpliado.pdf

Feld, V., Taussik I., Azaretto C. (2006). Test Procálculo. Test para la evaluación del procesamiento del número y el cálculo en niños. Buenos Aires: Paidós.

Fernández-Pinto, I., Santamaría, P., Sánchez-Sánchez, F., Carrasco, M. A., \& Del Barrio, V. (2015). Sistema de Evaluación de Niños y Adolescentes. SENA. Madrid: TEA Ediciones.

Ginsburg, H., Baroody, A. J., del Río, M. C. N., \& Guerra, I. L. (2007). TEMA 3: Test de competencia matemática básica. Madrid: TEA Ediciones.

Hambleton, R. K., Swaminathan, H. \& Rogers, H. J. (1991). Measurement methods for the social sciences series. Vol. 2. Fundamentals of item response theory. NY:

Sage Publications.

Instituto Nacional de Estadística y Censos (2012) Censo nacional de población, hogares y viviendas 2010: censo del Bicentenario : resultados definitivos, Serie B n 2. - 1a ed. Buenos Aires: Autor.

Kambanaros, M., Michaelides, M., \& Grohmann, K. K. (2015). Measuring word retrieval deficits in a multilingual child with SLI: Is there a better language? Journal of Neurolinguistics, 34, 112-130.

Korkman, M., Kirk, U., \& Kemp, S. (2007). NEPSY-II. Batería Neuropsicológica infantil. Madrid: Pearson.

Landis, J. R., \& Koch, G. G. (1977). The measurement of observer agreement for categorical data. Biometrics, 159-174.

Martínez Arias, R. (1996). Psicometría: teoría de los test psicológicos y educativos. Madrid: Síntesis.

Matute, E., Rosselli, M., Ardila, A. \& Ostrosky, F. (2007). Evaluación neurológica infantil (ENI-2). Ciudad de Mèxico: Manual moderno.

Novogrodsky, R., \& Friedmann, N. (2006). The production of relative clauses in syntactic SLI: A window to the nature of the impairment. Advances in Speech Language Pathology, 8(4), 364-375. 
Peyrin, C., Lallier, M., Demonet, J. F., Pernet, C., Baciu, M., Le Bas, J. F., \& Valdois, S. (2012). Neural dissociation of phonological and visual attention span disorders in developmental dyslexia: FMRI evidence from two case reports. Brain and language, 120(3), 381-394.

R Core Team (2013). R: A language and environment for statistical computing. R Foundation for Statistical Computing. Disponible en http://www.R-project.org/.

Reynolds, C. R. \& Bigler, E. D. (2001). TOMAL. Test de memoria y aprendizaje. Madrid: TEA Ediciones.

Rizopoulos, D. (2006). ltm: An R package for Latent Variable Modelling and Item Response Theory Analyses. Journal of Statistical Software. 17 (5). 1-25. Dispoible en http://www.jstatsoft.org/v17/i05/

Semel, E., Wiig, E.H. \& Secord, W. A. (2006). CELF-4. Clinical Evaluation of Language Fundamentals. Spanish Edition. United States of America: PsychCorp.

Singer, V., Cuadro, A., Costa, D., \& von Hagen, A. (2014). Evaluación de la eficacia de cálculo matemático: Manual Técnico del Test de Eficacia de Cálculo Matemático (TECA). Montevideo: Grupo Magro Ediciones. 


\section{ANEXOS}

\section{ANEXO A}

\section{Análisis de parametrización para las 75 pruebas de la BANEDI}

\section{Tabla A.1}

Análisis de parametrización para las pruebas del dominio de Memoria

\begin{tabular}{|c|c|c|c|c|c|c|}
\hline \multirow[t]{2}{*}{ Prueba } & \multirow[t]{2}{*}{$N$} & \multicolumn{2}{|c|}{$\begin{array}{l}\text { Índices de } \\
\text { dificultad }\end{array}$} & \multicolumn{2}{|c|}{$\begin{array}{c}\text { Índices de } \\
\text { discriminación }\end{array}$} & \multirow{2}{*}{$\begin{array}{l}\text { Marco } \\
\text { teórico }\end{array}$} \\
\hline & & $M$ & Rango & $M$ & Rango & \\
\hline 1.1 Lista de palabras $(n=4)$ & 951 & -0.34 & $-3.20-3.59$ & 2.99 & $1.90-3.71$ & TRI \\
\hline $1.2 \mathrm{El} \mathrm{mapa}(n=4)$ & 563 & -1.55 & $-3.10-0.98$ & 2.96 & $1.87-3.49$ & TRI \\
\hline $1.3 \mathrm{El} \mathrm{circo}$ & 951 & \multicolumn{4}{|c|}{ AP } & TCT \\
\hline 1.4 Lista de palabras II & 951 & \multicolumn{4}{|c|}{ AP } & TCT \\
\hline $1.5 \mathrm{El}$ mapa II & 563 & \multicolumn{4}{|c|}{ AP } & TCT \\
\hline $1.6 \mathrm{El}$ circo II & 870 & \multicolumn{4}{|c|}{ AP } & TCT \\
\hline $1.7 \mathrm{El}$ topo & 951 & \multicolumn{4}{|c|}{ AP } & TRI \\
\hline $1.8 \mathrm{El}$ topo II & 951 & \multicolumn{4}{|c|}{ NA } & NA \\
\hline 1.9 Animales & 951 & \multicolumn{4}{|c|}{ AP } & TRI \\
\hline 1.10 Animales II & 951 & \multicolumn{4}{|c|}{ NA } & NA \\
\hline
\end{tabular}

Nota. $N$ = cantidad de niños/adolescentes que completaron esta prueba; $M=$ Media; TCT = Teoría clásica de Test; $n=$ número de ítems en los que se basó en análisis; AP = Análisis en proceso; NA = No aplica este tipo de análisis.

\section{Tabla A.2}

Análisis de parametrización para las pruebas del dominio de Atención y Funciones ejecutivas

\begin{tabular}{|c|c|c|c|c|c|c|}
\hline \multirow[t]{2}{*}{ Prueba } & \multirow[t]{2}{*}{$N$} & \multicolumn{2}{|c|}{$\begin{array}{l}\text { Indices de } \\
\text { dificultad }\end{array}$} & \multicolumn{2}{|c|}{$\begin{array}{c}\text { Índices de } \\
\text { discriminación }\end{array}$} & \multirow{2}{*}{$\begin{array}{l}\text { Marco } \\
\text { teórico }\end{array}$} \\
\hline & & $M$ & Rango & $M$ & Rango & \\
\hline 2.1 Draco $(n=12)$ & 968 & -1.23 & $-1.24-(-1.22)$ & 1.03 & $1.01-1.04$ & TRI \\
\hline $2.2 \mathrm{El} \mathrm{barco}(n=13)$ & 968 & -0.70 & $-0.70-(-0.69)$ & 1.08 & $1.02-1.14$ & TRI \\
\hline $2.3 \mathrm{El}$ espacio $(n=30)$ & 968 & -1.14 & $-1.15-(-1.13)$ & 1.26 & $0.92-1.72$ & TRI \\
\hline 2.4 Nube $(n=70)$ & 968 & -0.70 & $-0.70-(-0.69)$ & 1.16 & $1.10-1.35$ & TRI \\
\hline 2.5 Números y personas $(n=24)$ & 870 & .47 & $.05-.91$ & .53 & $.17-.89$ & TCT \\
\hline 2.6 Colores al revés $(n=24)$ & 870 & .40 & $.01-.85$ & .44 & $.01-.87$ & TCT \\
\hline 2.7 Juegos & 184 & & & $\mathrm{~A}$ & & NA \\
\hline 2.8 Juegos II & 784 & & & A & & NA \\
\hline
\end{tabular}

Nota. $N=$ cantidad de niños/adolescentes que completaron esta prueba; $M=$ Media; TCT = Teoría clásica de Test; $n=$ número de ítems en los que se basó en análisis; NA = No aplica este tipo de análisis. 


\section{Tabla A.3}

Análisis de parametrización para las pruebas del dominio de Teoría de la Mente

\begin{tabular}{|c|c|c|c|c|c|c|}
\hline \multirow[t]{2}{*}{ Prueba } & \multirow[t]{2}{*}{$N$} & \multicolumn{2}{|c|}{$\begin{array}{l}\text { Índices de } \\
\text { dificultad }\end{array}$} & \multicolumn{2}{|c|}{$\begin{array}{c}\text { Índices de } \\
\text { discriminación }\end{array}$} & \multirow{2}{*}{$\begin{array}{l}\text { Marco } \\
\text { teórico }\end{array}$} \\
\hline & & $M$ & Rango & $M$ & Rango & \\
\hline 3.1 Caras $(n=12)$ & 198 & .81 & $.39-.98$ & .32 & $.01-.72$ & TCT \\
\hline 3.2 Sentimientos $(n=5)$ & 198 & -2.19 & $-3.97-(-0.41)$ & 1.45 & $0.96-1.81$ & TRI \\
\hline 3.3 Historias $(n=2)$ & 198 & -0.01 & $-2.09-2.57$ & 2.60 & $0.52-4.69$ & TRI \\
\hline 3.4 Historias II & 289 & & NA & & & TCT \\
\hline
\end{tabular}

Nota. $N=$ cantidad de niños/adolescentes que completaron esta prueba; $M=$ Media; TCT = Teoría clásica de Test; $n=$ número de ítems en los que se basó en análisis.

\section{Tabla A.4}

Análisis de parametrización para las pruebas del dominio de Habilidades metalingüísticas

\begin{tabular}{|c|c|c|c|c|c|c|}
\hline \multirow[t]{2}{*}{ Prueba } & \multirow[t]{2}{*}{$N$} & \multicolumn{2}{|c|}{$\begin{array}{l}\text { Índices de } \\
\text { dificultad }\end{array}$} & \multicolumn{2}{|c|}{$\begin{array}{c}\text { Índices de } \\
\text { discriminación }\end{array}$} & \multirow{2}{*}{$\begin{array}{l}\text { Marco } \\
\text { teórico }\end{array}$} \\
\hline & & $M$ & Rango & $M$ & Rango & \\
\hline 4.1 Unión de sonidos $(n=14)$ & 192 & .31 & $.01-.83$ & .43 & $.04-.82$ & TCT \\
\hline 4.2 Omisión de sonidos $(n=14)$ & 192 & .29 & $.21-.48$ & .78 & $.64-.93$ & TCT \\
\hline 4.3 Conteo de sonidos $(n=14)$ & 298 & .52 & $.30-.82$ & .79 & $.48-.95$ & TCT \\
\hline 4.4 Separación de sonidos $(n=14)$ & 192 & .64 & $.35-.91$ & .68 & $.25-.92$ & TCT \\
\hline
\end{tabular}

Nota. $N$ = cantidad de niños/adolescentes que completaron esta prueba; $M=$ Media; TCT = Teoría clásica de Test; $n=$ número de ítems en los que se basó en análisis. 


\section{Tabla A.5}

Análisis de parametrización para las pruebas del dominio de Lenguaje oral

\begin{tabular}{|c|c|c|c|c|c|c|}
\hline \multirow[t]{2}{*}{ Prueba } & \multirow[t]{2}{*}{$N$} & \multicolumn{2}{|c|}{$\begin{array}{l}\text { Índices de } \\
\text { dificultad }\end{array}$} & \multicolumn{2}{|c|}{$\begin{array}{c}\text { Índices de } \\
\text { discriminación }\end{array}$} & \multirow{2}{*}{$\begin{array}{l}\text { Marco } \\
\text { teórico }\end{array}$} \\
\hline & & $M$ & Rango & $M$ & Rango & \\
\hline 5.1 Palabras iguales y diferentes $(n=16)$ & 92 & .75 & $.46-.88$ & .38 & $.23-.56$ & TCT \\
\hline 5.2 Movimientos de la boca $(n=13)$ & 92 & .84 & $.43-.97$ & .32 & $.06-.93$ & TCT \\
\hline 5.3 Repetición de palabras $(n=12)$ & 92 & .86 & $.69-.94$ & .32 & $.01-.70$ & TCT \\
\hline 5.4 Repetición de palabras inventadas $(n=12)$ & 485 & .82 & $.65-.87$ & .38 & $.27-.67$ & TCT \\
\hline 5.5 Palabras incompletas $(n=12)$ & 485 & 67 & $.12-.96$ & .39 & $.09-.68$ & TCT \\
\hline $5.6 \mathrm{El}$ comedor $(n=8)$ & 303 & .84 & $.69-.94$ & .34 & $.16-.60$ & TCT \\
\hline $5.7 \mathrm{El}$ comedor II $(n=8)$ & 303 & .87 & $.69-.95$ & .30 & $.13-.61$ & TCT \\
\hline 5.8 Comidas, animales y profesiones & 985 & \multicolumn{4}{|c|}{ NA } & NA \\
\hline 5.9 Picnic $(n=8)$ & 485 & -0.19 & $-1.66-1.30$ & 1.72 & $1.10-2.30$ & TRI \\
\hline 5.10 Doble sentido $(n=6)$ & 595 & -0.12 & $-1.56-1.16$ & 2.42 & $1.58-4.35$ & TRI \\
\hline 5.11 Imágenes y frases $(n=6)$ & 198 & .81 & $.76-.85$ & .48 & $.40-.60$ & TCT \\
\hline 5.12 Palabras y frases $(n=9)$ & 787 & .79 & $.51-.92$ & .47 & $.22-.90$ & TCT \\
\hline 5.13 Frases incorrectas $(n=6)$ & 485 & .68 & $.57-.75$ & .74 & $.62-.88$ & TCT \\
\hline 5.14 Juegos, comidas y ropa & 485 & \multicolumn{4}{|c|}{ AP } & TCT \\
\hline 5.15 Situaciones $(n=6)$ & 985 & .85 & $.79-.95$ & .36 & $.14-.54$ & TCT \\
\hline 5.16 Comprensión del discurso $(n=6)$ & 390 & .79 & $.52-.90$ & 43 & $.23-.85$ & TCT \\
\hline
\end{tabular}

Nota. $N$ = cantidad de niños/adolescentes que completaron esta prueba; $M=$ Media; TCT = Teoría clásica de Test; $n=$ número de ítems en los que se basó en análisis; $\mathrm{AP}=$ Análisis en proceso; NA = No aplica este tipo de análisis. 


\section{Tabla A.6}

Análisis de parametrización para las pruebas del dominio de Habilidades visoespaciales

\begin{tabular}{|c|c|c|c|c|c|c|}
\hline \multirow[t]{2}{*}{ Prueba } & \multirow[t]{2}{*}{$N$} & \multicolumn{2}{|c|}{$\begin{array}{l}\text { Índices de } \\
\text { dificultad }\end{array}$} & \multicolumn{2}{|c|}{$\begin{array}{c}\text { Índices de } \\
\text { discriminación }\end{array}$} & \multirow{2}{*}{$\begin{array}{l}\text { Marco } \\
\text { teórico }\end{array}$} \\
\hline & & $M$ & Rango & $M$ & Rango & \\
\hline 6.1 Figuras entreveradas & 907 & \multicolumn{4}{|c|}{$\mathrm{AP}$} & TCT \\
\hline 6.2 Lápices & 432 & \multicolumn{4}{|c|}{ AP } & TCT \\
\hline 6.3 Vista desde arriba & 346 & \multicolumn{4}{|c|}{$\mathrm{AP}$} & TCT \\
\hline 6.4 Rompecabezas & 435 & \multicolumn{4}{|c|}{ AP } & TCT \\
\hline 6.5 Bloques & 907 & \multicolumn{4}{|c|}{ NA } & NA \\
\hline 6.6 Caminos & 744 & \multicolumn{4}{|c|}{$\mathrm{AP}$} & TRI \\
\hline 6.7 Dibujos $(n=4 / 6) \mathrm{a}$ & 774 & $-0.30 / 0.06 a$ & $-2.33-1.96$ & $1.71 / 2.71 \mathrm{a}$ & $1.43-3.09$ & TRI \\
\hline 6.8 Escritura normal & 718 & \multicolumn{4}{|c|}{ NA } & NA \\
\hline 6.9 Escritura rápida & 718 & \multicolumn{4}{|c|}{ NA } & NA \\
\hline 6.10 Fútbol $(n=12)$ & 718 & .73 & $.57-.94$ & .64 & $.15-.90$ & TCT \\
\hline
\end{tabular}

Nota. $N$ = cantidad de niños/adolescentes que completaron esta prueba; $M=$ Media; TCT = Teoría clásica de Test; $n=$ número de ítems en los que se basó en análisis; $\mathrm{AP}=$ Análisis en proceso; $\mathrm{NA}=$ No aplica este tipo de análisis. aValores para niños de 4 y 5 años y niños de 6 a 12 años. respectivamente.

\section{Tabla A.7}

Análisis de parametrización para las pruebas del dominio de Lenguaje escrito

\begin{tabular}{|c|c|c|c|c|c|c|}
\hline \multirow[t]{2}{*}{ Prueba } & \multirow[t]{2}{*}{$N$} & \multicolumn{2}{|c|}{$\begin{array}{l}\text { Índices de } \\
\text { dificultad }\end{array}$} & \multicolumn{2}{|c|}{$\begin{array}{c}\text { Índices de } \\
\text { discriminación }\end{array}$} & \multirow[t]{2}{*}{$\begin{array}{l}\text { Marco } \\
\text { teórico }\end{array}$} \\
\hline & & $M$ & Rango & $M$ & Rango & \\
\hline 7.1 Colores repetidos & 198 & \multicolumn{4}{|c|}{ NA } & NA \\
\hline 7.2 Letras repetidas & 784 & \multicolumn{4}{|c|}{ NA } & NA \\
\hline 7.3 Letras $(n=19)$ & 220 & .50 & $.34-.77$ & 90 & $.61-.99$ & TCT \\
\hline 7.4 Letras II $(n=6)$ & 82 & -0.97 & $-2.57-(-0.07)$ & 2.83 & $0.94-5.35$ & TRI \\
\hline 7.5 Lectura de palabras $(n=32)$ & 574 & \multicolumn{4}{|c|}{ AP } & TRI \\
\hline 7.6 Lectura de palabras inventadas $(n=32)$ & 574 & .82 & $.61-.98$ & .34 & $.04-.75$ & TCT \\
\hline 7.7 Velocidad lectora & 761 & \multicolumn{4}{|c|}{ NA } & NA \\
\hline 7.8 Preguntas sobre el texto $(n=3)$ a & 761 & -2.01 & $-7.21-1.53$ & 1.67 & $0.27-5.22$ & TRI \\
\hline 7.9 Dictado de palabras $(n=32)$ & 574 & .69 & $.39-.98$ & .62 & $.04-.91$ & TCT \\
\hline 7.10 Dictado de palabras inventadas $(n=32)$ & 574 & .71 & $.23-.95$ & .42 & $.09-.85$ & TCT \\
\hline 7.11 Cuento & 761 & \multicolumn{4}{|c|}{ AP } & TRI \\
\hline
\end{tabular}

Nota. $N=$ cantidad de niños/adolescentes que completaron esta prueba; $M=$ Media; TCT = Teoría clásica de Test; $n=$ número de ítems en los que se basó en análisis; AP = Análisis en proceso; $\mathrm{NA}=$ No aplica este tipo de análisis. aTres ítems diferentes para niños/adolescentes de $1^{\circ}$ Primaria. $2^{\circ}$ y $3^{\circ}$ Primaria. $4^{\circ}$ y $5^{\circ}$ Primaria y $6^{\circ}$ Primaria a $3^{\circ}$ Secundaria. respectivamente. 


\section{Tabla A.8}

Análisis de parametrización para las pruebas del dominio de Habilidades matemáticas

\begin{tabular}{|c|c|c|c|c|c|c|}
\hline \multirow[t]{2}{*}{ Prueba } & \multirow[t]{2}{*}{$N$} & \multicolumn{2}{|c|}{ Índices de dificultad } & \multicolumn{2}{|c|}{$\begin{array}{c}\text { Índices de } \\
\text { discriminación }\end{array}$} & \multirow{2}{*}{$\begin{array}{l}\text { Marco } \\
\text { teórico }\end{array}$} \\
\hline & & $M$ & Rango & $M$ & Rango & \\
\hline 8.1 Conteo & 217 & & $\mathrm{~N}$ & & & NA \\
\hline 8.2 Conteo al revés & 366 & & $\mathrm{~N}$ & & & NA \\
\hline 8.3 Conjuntos de puntos & 923 & & $\mathrm{Al}$ & & & TRI \\
\hline 8.4 Puntos y palabras-número & 923 & & $\mathrm{Al}$ & & & TRI \\
\hline 8.5 Puntos y números arábigos & 811 & & $\mathrm{Al}$ & & & TRI \\
\hline 8.6 Números arábigos & 811 & & $\mathrm{Al}$ & & & TRI \\
\hline 8.7 Dictado de números $(n=8 / 16)$ a & 536 & .78 & $.52-.97$ & .50 & $.06-1.00$ & TCT \\
\hline 8.8 Lectura de números $(n=16)$ & 536 & .87 & $.59-.98$ & .32 & $.03-1.00$ & TCT \\
\hline 8.9 Recta numérica & 811 & & Al & & & TCT \\
\hline 8.10 Cuentas con palabras-número & 706 & & $\mathrm{Al}$ & & & TRI \\
\hline 8.11 Cuentas con números arábigos & 706 & & $\mathrm{Al}$ & & & TRI \\
\hline 8.12 Cuentas escritas $(n=8 / 16) \mathrm{b}$ & 706 & .53 & $.02-.98$ & .57 & $.03-1.00$ & TCT \\
\hline 8.13 Problemas matemáticos $(n=6)_{\mathrm{c}}$ & 811 & $-0.33 /-0.46 c$ & $-2.57-2.00$ & $1.84 / 1.90_{\mathrm{c}}$ & $1.19-2.96$ & TRI \\
\hline
\end{tabular}

Nota. $N=$ cantidad de niños/adolescentes que completaron esta prueba; $M=$ Media; TCT = Teoría clásica de Test; $n=$ número de ítems en los que se basó en análisis; AP = Análisis en proceso; NA = No aplica este tipo de análisis. aValores para niños de $1^{\circ}$ y $2^{\circ}$ Primaria y niños/adolescentes de $3^{\circ}$ a $6^{\circ}$ Primaria, respectivamente. bItems diferentes para niños/adolescentes de $1^{\circ}$ y $2^{\circ}$ Primaria, $3^{\circ}$ Primaria, $4^{\circ}$ Primaria, $5^{\circ}$ Primaria, $6^{\circ}$ Primaria y $1^{\circ}$ a $3^{\circ}$ Secundaria, respectivamente; cItems diferentes para niños/adolescentes de Preescolar Nivel 5 a $2^{\circ}$ Primaria y $3^{\circ}$ Primaria a $3^{\circ}$ Secundaria, respectivamente. 


\section{ANEXO B}

\section{Análisis de varianza (ANOVA) para las 75 pruebas de la BANEDI}

\section{Tabla B.1}

Efecto de la variable edad sobre los puntajes directos de las pruebas del dominio de Memoria en base a Análisis de varianza (ANOVA)

\begin{tabular}{|c|c|c|c|c|}
\hline Prueba & Edades de aplicación & $F$ & $g l$ & $p$ \\
\hline 1.1 Lista de palabras & 4 a 15 años & 1157.0 & 1,949 & $<.001 * * *$ \\
\hline $1.2 \mathrm{El} \mathrm{mapa}$ & 4 a 9 años & 220.9 & 1,561 & $<.001 * * *$ \\
\hline $1.3 \mathrm{El} \mathrm{circo}$ & 4 a 15 años & 772.3 & 1,949 & $<.001 * * *$ \\
\hline 1.4 Lista de palabras II & 4 a 15 años & 869.7 & 1,949 & $<.001 * * *$ \\
\hline $1.5 \mathrm{El}$ mapa II & 4 a 9 años & 152.4 & 1,561 & $<.001 * * *$ \\
\hline $1.6 \mathrm{El}$ circo II & 5 a 15 años & 817.2 & 1,949 & $<.001 * * *$ \\
\hline 1.7 El topo & 4 a 15 años & 2.2 & 1,949 & .133 \\
\hline $1.8 \mathrm{El}$ topo II & 4 a 15 años & & NA & \\
\hline 1.9 Animales & 4 a 15 años & 39.8 & 1,949 & $<.001 * * *$ \\
\hline 1.10 Animales II & 4 a 15 años & & NA & \\
\hline
\end{tabular}

Nota. NA = No aplica; $g l=$ grados de libertad; $* * * p<.001$ (bilateral).

\section{Tabla B.2}

Efecto de la variable edad sobre los puntajes directos de las pruebas del dominio de Atención y Funciones ejecutivas en base a Análisis de varianza (ANOVA)

\begin{tabular}{lcccc}
\hline \multicolumn{1}{c}{ Prueba } & Edades de aplicación & $F$ & $g l$ & $p$ \\
\hline 2.1 Dracoa & 4 a 15 años & 1142.0 & 1,966 & $<.001 * * *$ \\
2.2 El barcoa & 4 a 15 años & 16.2 & 1,940 & $<.001 * * *$ \\
2.3 El espacioa & 4 a 15 años & 736.5 & 1,966 & $<.001 * * *$ \\
2.4 Nubea & 4 a 15 años & 89.9 & 1,966 & $<.001 * * *$ \\
2.5 Números y personas & 5 a 15 años & 599.9 & 1,868 & $<.001 * * *$ \\
2.6 Colores al revés & 4 a 15 años & 893.2 & 1,868 & $<.001 * * *$ \\
2.7 Juegos & 4 y 5 años & 15.16 & 1,182 & $<.001 * * *$ \\
2.7 Juegos II & 6 a 15 años & 147.0 & 1,782 & $<.001 * * *$ \\
\hline Nota. aAnálisis en base a tiempos de reacción; $g l=$ grados de libertad; *** $p<.001$ (bilateral).
\end{tabular}

\section{Tabla B.3}

Efecto de la variable edad sobre los puntajes directos de las pruebas del dominio de Teoría de la Mente en base a Análisis de varianza (ANOVA)

\begin{tabular}{|c|c|c|c|c|}
\hline Prueba & Edades de aplicación & $F$ & $g l$ & $p$ \\
\hline 3.1 Caras & 4 y 5 años & 24.0 & 1,196 & $<.001 * * *$ \\
\hline 3.2 Sentimientos & 4 y 5 años & 12.7 & 1,196 & $<.001 * * *$ \\
\hline 3.3 Historias & 4 y 5 años & 7.3 & 1,196 & $<.01 * *$ \\
\hline 3.4 Historias II & 6 a 8 años & 9.5 & 1,287 & $<.01^{* * *}$ \\
\hline
\end{tabular}

Nota. $g l=$ grados de libertad; $* * * p<.001$ (bilateral); $* * p<.01$ (bilateral). 


\section{Tabla B.4}

Efecto de la variable edad sobre los puntajes directos de las pruebas del dominio de Habilidades Metalingüísticas en base a Análisis de varianza (ANOVA)

\begin{tabular}{lcccc}
\hline \multicolumn{1}{c}{ Prueba } & Edades de aplicación & $F$ & $g l$ & $p$ \\
\hline 4.1 Unión de sonidos & 6 y 7 años & 15.0 & 1,190 & $<.001^{* * *}$ \\
4.2 Omisión de sonidos & 6 y 7 años & 25.6 & 1,190 & $<.001^{* * *}$ \\
4.3 Conteo de sonidos & 5 a 7 años & 135.5 & 1,296 & $<.001^{* * *}$ \\
4.4 Separación de sonidos & 6 y 7 años & 16.8 & 1,190 & $<.001^{* * *}$ \\
\hline
\end{tabular}

Nota. $g l=$ grados de libertad; $* * * p<.001$ (bilateral).

\section{Tabla B.5}

Efecto de la variable edad sobre los puntajes directos de las pruebas del dominio de

Lenguaje oral en base a Análisis de varianza (ANOVA)

\begin{tabular}{|c|c|c|c|c|}
\hline Prueba & Edades de aplicación & $F$ & $g l$ & $p$ \\
\hline 5.1 Palabras iguales y diferentes & 4 años & & $\mathrm{NA}$ & \\
\hline 5.2 Movimientos de la boca & 4 años & & NA & \\
\hline 5.3 Repetición de palabras & 4 años & & NA & \\
\hline 5.4 Repetición de palabras inventadas & 4 a 8 años & 120.5 & 1,483 & $<.001 * * *$ \\
\hline 5.5 Palabras incompletas & 4 a 8 años & 213.5 & 1,483 & $<.001 * * *$ \\
\hline $5.6 \mathrm{El}$ comedor & 4 a 6 años & 35.3 & 1,301 & $<.001^{* * *}$ \\
\hline $5.7 \mathrm{El}$ comedor II & 4 a 6 años & 59.0 & 1,388 & $<.001 * * *$ \\
\hline 5.8 Comidas, animales y profesiones & 4 a 15 años & 1389.0 & 1,983 & $<.001 * * *$ \\
\hline 5.9 Picnic & 4 a 8 años & 389.4 & 1,483 & $<.001 * * *$ \\
\hline 5.10 Doble sentido & 8 a 15 años & 231.8 & 1,593 & $<.001 * * *$ \\
\hline 5.11 Imágenes y frases & 4 y 5 años & 2.5 & 1,196 & $.11 \mathrm{~ns}$ \\
\hline 5.12 Palabras y frases & 6 a 15 años & 284.2 & 1,785 & $<.001 * * *$ \\
\hline 5.13 Frases incorrectas & 5 a 8 años & 130.8 & 1,391 & $<.001 * * *$ \\
\hline 5.14 Juegos, comidas y ropa & 4 a 8 años & 137.3 & 1,483 & $<.001 * * *$ \\
\hline 5.15 Situaciones & 4 a 15 años & 305.7 & 1,983 & $<.001 * * *$ \\
\hline 5.16 Comprensión del discurso & 4 a 7 años & 117.5 & 1,388 & $<.001 * * *$ \\
\hline
\end{tabular}

Nota. NA = No aplica; $g l=$ grados de libertad; $* * * p<.001$ (bilateral); nsno llega a valores estadísticamente significativos. 


\section{Tabla B.6}

Efecto de la variable edad y año escolar sobre los puntajes directos de las pruebas del dominio de Habilidades viso-espaciales en base a Análisis de varianza (ANOVA)

\begin{tabular}{|c|c|c|c|c|}
\hline Prueba & Edades/año escolar de aplicación & $F$ & $g l$ & $p$ \\
\hline 6.1 Figuras entreveradas & 4 a 8 años & 149.7 & 1,430 & $<.001 * * *$ \\
\hline 6.1 Figuras entreveradas & 9 a 15 años & 39.6 & 1,473 & $<.001 * * *$ \\
\hline 6.2 Lápices & 4 a 8 años & 145.8 & 1,430 & $<.001 * * *$ \\
\hline 6.3 Vista desde arriba & 4 a 7 años & 20.6 & 1,344 & $<.001 * * *$ \\
\hline 6.4 Rompecabezas & 6 a 10 años & 114.5 & 1,433 & $<.001 * * *$ \\
\hline 6.5 Bloques & 5 a 15 años & 48.9 & 1,819 & $<.001 * * *$ \\
\hline 6.6 Caminos & 4 a 12 años & 134.0 & 1,772 & $<.001 * * *$ \\
\hline 6.7 Dibujos & 4 y 5 años & 9.8 & 1,187 & $<.01 * *$ \\
\hline 6.7 Dibujos & 6 a 12 años & 115.6 & 1,583 & $<.001 * * *$ \\
\hline 6.8 Escritura normal & $1^{\circ}$ Primaria a $3^{\circ}$ Secundaria & 896.2 & 1,716 & $<.001 * * *$ \\
\hline 6.9 Escritura rápida & $1^{\circ}$ Primaria a $3^{\circ}$ Secundaria & 1021.0 & 1,716 & $<.001^{* * *}$ \\
\hline 6.10 Fútbol & 6 a 15 años & 266.8 & 1,716 & $<.001^{* * *}$ \\
\hline
\end{tabular}

Nota. NA = No aplica; $g l=$ grados de libertad; ***p $<.001$ (bilateral); $* * p<.01$ (bilateral).

\section{Tabla B.7}

Efecto de la variable edad y el año escolar sobre los puntajes directos de las pruebas del dominio de Lenguaje escrito en base a Análisis de varianza (ANOVA)

\begin{tabular}{|c|c|c|c|c|}
\hline Prueba & Edades/año escolar de aplicación & $F$ & $g l$ & $p$ \\
\hline 7.1 Colores repetidosa & 4 y 5 años & 12.77 & 1,196 & $<.001 * * *$ \\
\hline 7.2 Letras repetidasa & 6 a 15 años & 536.9 & 1,782 & $<.001 * * *$ \\
\hline 7.3 Letras & Preescolar Nivel 4 y 5 & 57.29 & 1,218 & $<.001 * * *$ \\
\hline 7.4 Letras II & $1^{\circ}$ Primaria & & NA & \\
\hline 7.5 Lectura de palabrasa & $1^{\circ}$ a $6^{\circ}$ Primaria & 262.3 & 1,572 & $<.001 * * *$ \\
\hline 7.6 Lectura de palabras inventadasa & $1^{\circ}$ a $6^{\circ}$ Primaria & 218.4 & 1,572 & $<.001 * * *$ \\
\hline 7.7 Velocidad lectora & $1^{\circ}$ Primaria a $3^{\circ}$ Secundaria & 1315.0 & 1,759 & $<.001 * * *$ \\
\hline 7.8 Preguntas sobre el texto & $2^{\circ}$ a $3^{\circ}$ Primaria & 12.47 & 1,178 & $<.001 * * *$ \\
\hline 7.8 Preguntas sobre el texto & $4^{\circ}$ a $5^{\circ}$ Primaria & 5.8 & 1,200 & $<.05 *$ \\
\hline 7.8 Preguntas sobre el texto & $6^{\circ}$ Primaria a $3^{\circ}$ Secundaria & 0.1 & 1,272 & $.97 \mathrm{~ns}$ \\
\hline 7.9 Dictado de palabras & $1^{\circ}$ a $6^{\circ}$ Primaria & 816.5 & 1,572 & $<.001 * * *$ \\
\hline 7.10 Dictado de palabras inventadas & $1^{\circ}$ a $6^{\circ}$ Primaria & 387.0 & 1,572 & $<.001 * * *$ \\
\hline 7.11 Cuento & $1^{\circ}$ Primaria a $3^{\circ}$ Secundaria & 257.0 & 1,759 & $<.001 * * *$ \\
\hline
\end{tabular}

(bilateral); $* p<.05$ (bilateral); nsno llega a valores estadísticamente significativos. 


\section{Tabla B.8}

Efecto de la variable año escolar sobre los puntajes directos de las pruebas del

dominio de Habilidades matemáticas en base a Análisis de varianza (ANOVA)

\begin{tabular}{|c|c|c|c|c|}
\hline Prueba & Edades de aplicación & $F$ & $g l$ & $p$ \\
\hline 8.1 Conteo & Preescolar Nivel 4 & & NA & \\
\hline 8.2 Conteo al revés & Preescolar Nivel 5 a $3^{\circ}$ Primaria & 10.21 & 1,364 & $<.001 * * *$ \\
\hline 8.3 Conjuntos de puntosa & Preescolar Nivel 4 a $3^{\circ}$ Secundaria & 220.2 & 1,921 & $<.001 * * *$ \\
\hline 8.4 Puntos y palabras-númeroa & Preescolar Nivel 4 a $3^{\circ}$ Secundaria & 8.7 & 1,921 & $<.01 * *$ \\
\hline 8.5 Puntos y números arábigosa & Preescolar Nivel 5 a $3^{\circ}$ Secundaria & 673.4 & 1,809 & $<.001 * * *$ \\
\hline 8.6 Números arábigosa & Preescolar Nivel 4 a $3^{\circ}$ Secundaria & 881.4 & 1,809 & $<.001^{* * * *}$ \\
\hline 8.7 Dictado de números & $1^{\circ}$ a $6^{\circ}$ Primaria & 1256.0 & 1,534 & $<.001^{* * * *}$ \\
\hline 8.8 Lectura de números & $1^{\circ}$ a $6^{\circ}$ Primaria & 576.4 & 1,704 & $<.001^{* * *}$ \\
\hline 8.9 Recta numérica & Preescolar Nivel 5 a $3^{\circ}$ Secundaria & 747.1 & 1,809 & $<.001 * * *$ \\
\hline 8.10 Cuentas con palabras-númeroa & $1^{\circ}$ Primaria a $3^{\circ}$ Secundaria & 574.6 & 1,704 & $<.001^{* * * *}$ \\
\hline 8.11 Cuentas con números arábigosa & $1^{\circ}$ Primaria a $3^{\circ}$ Secundaria & 263.8 & 1,704 & $<.001^{* * * *}$ \\
\hline 8.12 Cuentas escritas & $1^{\circ}$ Primaria a $3^{\circ}$ Secundaria & & NA & \\
\hline 8.13 Problemas matemáticos & Preescolar Nivel 5 a $3^{\circ}$ Secundaria & 1854.0 & 1,809 & $<.001 * * *$ \\
\hline
\end{tabular}

Nota. NA $=$ No aplica. aAnálisis en base a tiempos de reacción. $g l=$ grados de libertad; $* * * p<.001$

(bilateral); ** $p<.01$ (bilateral). 